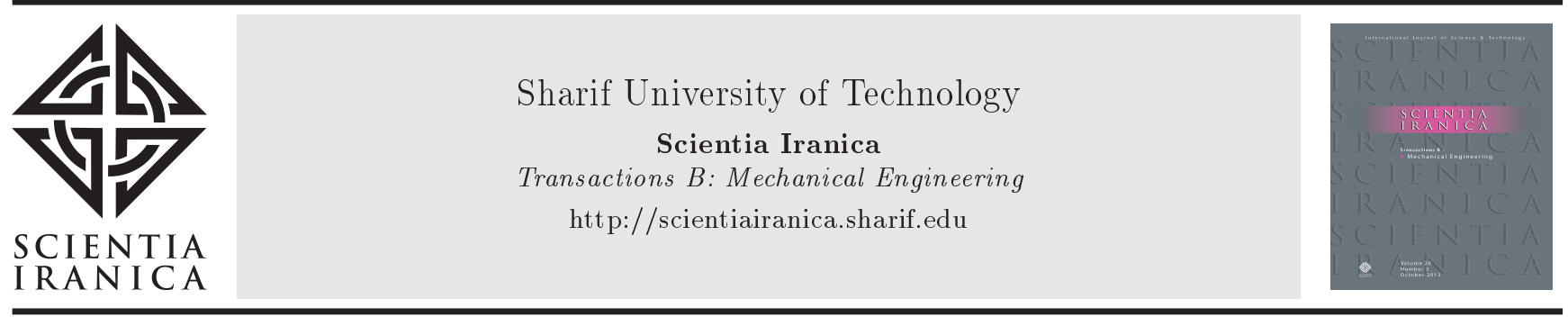

\title{
Free vibration analysis of functionally graded stiffened micro-cylinder based on the modified couple stress theory
}

\author{
S. Jabbarian and M.T. Ahmadian* \\ School of Mechanical Engineering, Sharif University of Technology, Tehran, Iran.
}

Received 4 December 2016; received in revised form 24 May 2017; accepted 28 August 2017

\author{
KEYWORDS \\ Free vibration; \\ Micro-cylinder; \\ Functionally graded \\ material; \\ Stiffened; \\ Modified couple \\ stress.
}

\begin{abstract}
In this paper, free vibration of the micro-cylinder made by functionally graded material stiffened in circumferential direction was investigated based on modified couple stress and first-order shear deformation theories. Modified Couple Stress Theory (MCST) was used to catch size effects in micro scales. By using first-order shear deformation theory and Hamilton's principle, the general equations of motion and corresponding boundary conditions were derived. Free vibration of the structure was investigated by implementing simply-supported boundary condition as a common case. The effects of different parameters, such as dimensionless length scale parameter, distribution of FGM properties, number of stiffeners, thickness and length on the natural frequencies, were calculated and compared with those of classical continuum theory. Results show that effects of the size are considerable; moreover, using stiffeners leads to an increase in natural frequencies due to an increase in stiffness of the cylinder.
\end{abstract}

(C) 2018 Sharif University of Technology. All rights reserved.

\section{Introduction}

Cylindrical shells specially stiffened by rings are important and useful parts of different industries and have a wide range of applications in various fields of engineering from aerospace, marine vessels to pressurized water reactors. Such a high number of applications makes the analysis of these structures so important; further, due to the usage of these parts in dynamic conditions, research made on the dynamic characteristics and their vibrational behavior has much more importance.

Many models of thin shell cylinders and stiffened ones have been presented by researchers since 1950's

*. Corresponding author. Tel./Fax: 982166165503 E-mail addresses: siavash.jabbarian@gmail.com (S. Jabbarian); ahmadian@sharif.edu (M.T. Ahmadian)

doi: $10.24200 /$ sci.2017.4348 in both micro and macro scales which simulate the behavior of such structures.

Basdekas and Chi [1] investigated the dynamic response of an oddly stiffened cylindrical shell structure. In the formulation, the shell was allowed to have any prescribed thickness variation. In order to have concentrated masses, any number of rings of arbitrary non-uniform cross-sections and spacing should be attached either to the shell or to the stiffening ring. Modified variational method was used as a method of solution. Displacement is assumed representable by a series of the products of the normal modes of a simple uniform shell and unknown functions which are only dependent on time. This paper explains how the resulting formulation can be utilized to solve the related problems such as static response, determination of natural frequencies, elastic stability, etc.

Zhou [2] investigated vibration and stability of ring-stiffened thin-walled cylindrical shells conveying fluid. By using Flügge shell theory and Hamilton's 
principle, equations of motion of ring-stiffened thinwalled cylindrical shells conveying fluid were derived. Effects of fluid velocity, Young modulus, the size and the number of stiffeners on natural frequency, and the instability characteristics were examined.

Hoppmann [3] researched the free vibration of simply-supported stiffened cylindrical shells by analytical and experimental methods and introduced an analytical method for shells stiffened by equal strength rings. Mikulas and McElman [4] investigated the free vibration of eccentrically stiffened simply-supported cylindrical shells. To smear the effects of stiffeners, they used averaging method, and found that eccentricity has remarkable effect on the free vibration of stiffened shells and the consequent natural frequencies.

After the production of Functionally Graded Materials (FGMs) in the middle of $80 \mathrm{~s}$, their wide applications have emerged in a variety of industries due to their special material properties that vary continuously and smoothly through certain dimensions. FGMs are made by combining two or more materials using four different methods such as centrifugal, metallurgy powder methods. Typically, these materials are made from a mixture of ceramics and metal in which the ceramic component provides high-temperature resistance due to its low thermal conductivity, while the metal component prevents fracture due to thermal stress. From a micro-scale perspective, having unique properties, such as high stiffness and great thermal resistance capacity, has led to the wide applications of such materials in aerospace, nuclear plants, and other engineering applications, to name a few [5-10].

According to previous laboratory researches, the classical continuum theory is unable to capture size effects in the examination of the behavior of micro structures. Thus, in recent years, non-classical continuum theories, such as strain gradient theory and couple stress theory, have been used to study micro scale structures.

In 1960s, some researchers introduced the couple stress elasticity theory [11-13]. In this theory, some higher-order material length scale parameters appear, in addition to the two classical Lame constants. Yang et al. [14] developed a modified couple stress theory by considering an extra equilibrium equation besides the classical equilibrium equations of forces and moments of forces where this additional equation is the equilibrium of moments of couples. Then, they concluded that this additional equilibrium equation implies the symmetry of the couple stress tensor. Accordingly, they modified the constitutive equations of the couple stress theory and presented new constitutive equations.

Zeighampour and Tadi Beni [15] modelled cylindrical thin shell based on the modified strain gradient theory. Size effects were considered through the modified strain gradient theory. By implementing
Hamilton's principle, partial equations of motion with classical and non-classical corresponding boundary conditions were derived. Finally, the free vibration of the single-walled carbon nanotube (SWCNT) as an example was investigated.

Zhou et al. [16] studied free vibration of micro and nano shells based on the modified couple stress theory. By introducing one internal material length scale parameter, a new dynamic model is derived based on the modified couple stress theory. After implementing energy approach, Lagrange equations of motion are derived and the natural frequency is analytically solved. They showed that the micro-scale shell has some essential vibration characteristics, and it is shown that, using this model, the natural frequencies are size dependent for a micro-scale shell which is simply supported at both ends.

Hossein-Hashemi et al. [17] investigated free vibrations of size-dependent closed micro/nano spherical shell based on the modified couple stress theory and application of first-order shear deformation theory. The governing equations of motion were derived by implementing Hamilton's principle; in addition, by using the generalized differential quadrature method, these equations were solved. The effects of changing geometrical and scale parameters on the natural frequency were investigated. It was shown that the scale parameter is extremely effective in the natural frequency of the micro/nano sphere. This issue is bolder in the thick spherical shell.

There are also many researches about using the modified couple stress theory in beams [18-23]. For example, by utilizing the modified couple stress theory, Park and Gao [18] analyzed the static mechanical properties of an Euler-Bernoulli beam. Kong et al. [19] derived the governing equation, initial and boundary conditions of an Euler-Bernoulli beam based on the modified coupled stress theory using the Hamilton's principle.

In addition, there are many researches focused on investigating vibrational behavior of micro and nano structures, especially cylindrical shells of functionally graded materials. Jomehzadeh et al. [24] examined the bending of a part of FGM annular sector plates using the first-order shear deformation plate theory; as the structure's dimensions diminish in the micro scale, the effect of structure size plays a significant role in the correct examination of such structures in minute scales. In addition, Asghari et al. $[25,26]$ investigated the sizedependent behavior of FGM micro beams using the modified couple stress theory and the Euler-Bernoulli and Timoshenko beam models. Sadeghi et al. [27] examined strain gradient elasticity formulation to analyze FGM micro-cylinders. The material properties are considered as a function of power low in radial direction. The governing differential equation was 
derived as a fourth-order ODE. It was depicted in the paper that the characteristic length parameter has considerable effect on the stress distribution of FGM micro-cylinders. In addition, it is shown that the FGM power index has an important effect on the maximum radial and tangential stresses.

Sahmani et al. [28] investigated size-dependent dynamic stability response of higher-order shear deformable cylindrical micro shells made of functionally graded materials. Simply supported ends were considered for this problem. Material properties of the micro shells vary in the thickness direction according to the Mori-Tanaka theory. The modified couple stress and the classical higher-order shear deformation shell theories were used to develop a non-classical shell model. By using Hamilton's principle, differential equations of motion and corresponding boundary conditions were derived. It was shown that, with the decrease of the value of dimensionless length scale parameter, the width of the instability region for an FGM micro shell increased. In addition, it was shown that the classical shell model has an overestimated prediction concerning the width of instability region corresponding to the FGM micro shells, especially with lower values of material property gradient index.

Bedroud et al. [29] examined the buckling of FGM circular/annular nanoplates under uniform inplane radial compressive load with a concentric internal ring support. The material properties vary according to a power-law distribution of the volume fraction of the constituents, whereas Poison's ratio is set to be constant. To solve this problem, the circular/annular nanoplate was divided into an annular segment and a core circular/annular segment at the location of the internal ring. Solution for two segments was combined with interfacial conditions. It was demonstrated that the ring increases the buckling capacity. In addition, effects of small scales on the maximum buckling load for different parameters, such as radius and thickness of FGM nanoplate, boundary conditions, mode numbers, and material properties, were investigated.

In this paper, the free vibration of micro-cylinder made of the functionally graded materials, stiffened in circumferential direction, has been investigated based on the modified couple stress and first-order shear deformation theories. Material properties of the FGM shell have been assumed as continuously variable along thickness. The Modified Couple Stress Theory (MCST) has been utilized to capture size effects in micro scales; by considering first-order shear deformation theory and using Hamilton's principle, the general equations of motion and corresponding boundary conditions have been derived. Finally, as a common case, simply supported boundary condition has been considered, and free vibration of functionally graded stiffened micro-cylinder has been investigated. The

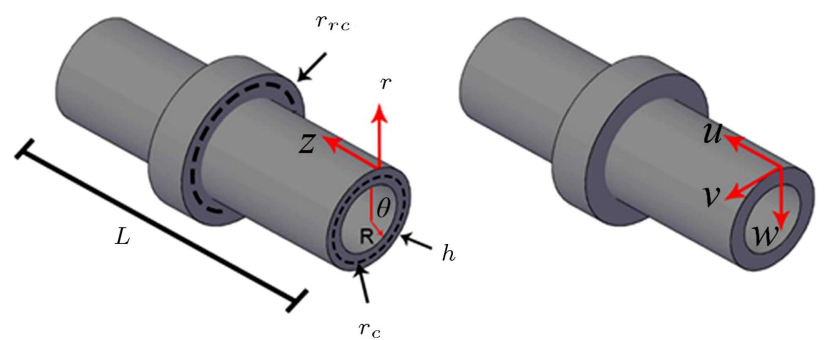

Figure 1. Schematic of stiffened FGM micro-cylinder.

effects of different parameters, such as dimensionless length scale parameter, distribution of FGM properties, number of stiffeners, and thickness and length on the natural frequencies, have been calculated and compared with the effect of classical continuum theory.

\section{Theoretical formulation}

In this paper, as illustrated by Figure 1, the FGM cylindrical shell is shown with length $L$, radius $R$, and thickness $h$. The shell circumferentially stiffened by $N$ rings though one ring is shown in the figure, and the space between the rings and depth of each ring may vary along the cylindrical shell.

\subsection{Functionally graded materials}

FGM is usually made by the combination of two components (e.g., ceramics and metal), and the material properties of the FGM cylindrical shell vary continuously and consistently from those of ceramics on the inner surface of the cylindrical shell to the properties of the metal on the outer surface as a function of constituent's volume fraction. Variation in volume fraction of metal and ceramics, according to power law distribution along cylindrical shell thickness, is expressed in the following equations:

$$
\begin{aligned}
V_{c} & =\left(\frac{2 \hat{r}+h}{2 h}\right)^{N}, \\
V_{m} & =1-V_{c},
\end{aligned}
$$

where $N$ stands for power index, which varies at $0 \leq$ $N \leq \infty$ interval, and as illustrated by Figure $1, \hat{r}$ stands for the arbitrary surface distance from the inner ones of the cylindrical shell. Therefore, the material properties of this cylindrical shell are expressed as:

$$
\begin{aligned}
& E(\hat{r})=\left(E_{c}-E_{m}\right)\left(\frac{2 \hat{r}+h}{2 h}\right)^{N}+E_{m}, \\
& \rho(\hat{r})=\left(\rho_{c}-\rho_{m}\right)\left(\frac{2 \hat{r}+h}{2 h}\right)^{N}+\rho_{m}, \\
& \nu(\hat{r})=\left(\nu_{c}-\nu_{m}\right)\left(\frac{2 \hat{r}+h}{2 h}\right)^{N}+\nu_{m},
\end{aligned}
$$

where $E_{m}, \rho_{m}$, and $\nu_{m}$ are obtained from $\hat{r}=-h / 2$, 
and $E_{c}, \rho_{c}$ and $v_{c}$ are obtained from $\hat{r}=h / 2$, which, respectively, represent Young's modulus, density, and Poisson's ratio of metal and ceramics. It should be noted that the length scale parameter herein is assumed constant in FGM shell.

\subsection{Modified couple stress}

In the modified couple stress theory, the strain energy density for a linear elastic material in infinitesimal deformation is written as follows [8]:

$$
U=\frac{1}{2} \int_{\Omega}\left(\sigma_{i j} \varepsilon_{i j}+m_{i j} \chi_{i j}\right) d V,
$$

where:

$$
\begin{aligned}
\varepsilon_{i j} & =\frac{1}{2}\left(u_{i, j}+u_{j, i}\right), \\
\chi_{i j} & =\frac{1}{4}\left(e_{i p q} \eta_{j p q}+e_{j p q} \eta_{i p q}\right), \\
\sigma_{i j} & =C_{i j k l} \varepsilon_{k l}, \\
m_{i j} & =2 l^{2} \mu(\hat{z}) \chi_{i j},
\end{aligned}
$$

in which $\sigma_{i j}, \varepsilon_{i j}, m_{i j}, \eta_{i j}$, and $\chi_{i j}$ denote the components of the symmetric parts of stress tensor, $\sigma$, strain tensor, $\boldsymbol{\varepsilon}$, the deviatoric part of couple stress tensor, $\mathbf{m}$, deviatoric stretch gradient tensor, and the symmetric part of curvature tensor $\chi$, respectively; $u_{i, j}$ is the component of displacement vector.

In the above equation, $l$ is the material length scale parameter, which reflects the effect of couple stress that is related to symmetric rotation gradient.

\subsection{First-order shear deformation theory}

This theory assumes that there is a linear variation of displacement across the plate thickness; yet, the plate thickness does not change during deformation. An additional assumption is that the normal stress through the thickness is ignored, which is called plane stress condition.

The stress tensor can be derived as follows:

$$
\begin{aligned}
& \left\{\begin{array}{l}
\sigma_{z z} \\
\sigma_{\theta \theta} \\
\sigma_{z \theta}
\end{array}\right\}=\left[\begin{array}{ccc}
C_{11} & C_{12} & 0 \\
C_{21} & C_{22} & 0 \\
0 & 0 & C_{33}
\end{array}\right]\left\{\begin{array}{l}
\varepsilon_{z z} \\
\varepsilon_{\theta \theta} \\
2 \varepsilon_{z \theta}
\end{array}\right\}, \\
& \left\{\begin{array}{l}
\sigma_{r z} \\
\sigma_{r \theta}
\end{array}\right\}=\left[\begin{array}{cc}
C_{44} & 0 \\
0 & C_{55}
\end{array}\right]\left\{\begin{array}{l}
2 \varepsilon_{r z} \\
2 \varepsilon_{r \theta}
\end{array}\right\}, \\
& C_{11}=C_{22}=\frac{E(\hat{r})}{1-\nu(\hat{r})^{2}}, \quad C_{12}=\frac{E(\hat{r}) \nu(\hat{r})}{1-\nu(\hat{r})^{2}}, \\
& C_{33}=C_{44}=C_{55}=\mu(\hat{r}),
\end{aligned}
$$

where $E(\hat{r}), \nu(\hat{r})$, respectively, represent Young's modulus and Poisson's ratio of the FGM cylindrical shell.
As displayed by Figure 1, the displacement field of cylindrical shell and ring based on the first-order shear deformation theory along the three directions of $r, \theta$, and $z$ is expressed as follows [8]:

$$
\begin{aligned}
& z: u(z, r, \theta, t)=U(z, \theta, t)+r \psi_{z}(z, \theta, t) \\
& \theta: \nu(z, r, \theta, t)=V(z, \theta, t)+r \psi_{\theta}(z, \theta, t) \\
& r: w(z, r, \theta, t)=W(z, \theta, t) \\
& z: u(r, \theta, t)=U(\theta, t)+r^{\prime} \psi_{z}(\theta, t) \\
& \theta: \nu(r, \theta, t)=V(\theta, t)+r^{\prime} \psi_{\theta}(\theta, t) \\
& r: w(r, \theta, t)=W(\theta, t)
\end{aligned}
$$

where $U(z, \theta, t), V(z, \theta, t)$, and $W(z, \theta, t)$ are the neutral axis displacements of the cylinder, and $\psi_{\theta}(z, \theta, t)$, and $\psi_{z}(z, \theta, t)$ are rotations of a transverse normal about the axial and circumferential directions. Also, $U(\theta, t), V(\theta, t)$, and $W(\theta, t)$ are the neutral axis displacements, and $\psi_{\theta}(\theta, t)$ and $\psi_{z}(\theta, t)$ are rotations of a transverse normal about the axial and circumferential directions for the ring. Displacements in $z$ direction for ring have been neglected due to the short height of the ring in comparison with its cylinder length.

Position of the neutral axis is expressed as follows:

$$
\int_{A} \sigma_{z z} d A=\int_{A} \frac{E(\hat{r})}{1-\nu^{2}(\hat{r})}\left(r \frac{\partial^{2} W}{\partial z^{2}}\right) d A=0,
$$

where $r=\hat{r}-\hat{r}_{c}$, by replacing this relation into Eq. (9):

$$
\begin{aligned}
r_{c}= & \frac{\int_{A} \frac{E(\hat{r})}{1-\nu^{2}(\hat{r})} \hat{r} d A}{\int_{A} \frac{E(\hat{r})}{1-\nu^{2}(\hat{r})} d A}, \\
r_{r c}= & \frac{\int_{\text {ring }} \frac{E_{\text {ring }}(\hat{r})}{1-\nu_{\text {ring }}^{2}(\hat{r})} \hat{r} d A_{\text {ring }}}{\int_{A_{\text {ring }}} \frac{E_{\text {ring }}(\hat{r})}{1-\nu_{\text {ring }}^{2}(\hat{r})} d A_{\text {ring }}},
\end{aligned}
$$

in which $\hat{r}_{c}$ and $\hat{r}_{r c}$ are respectively the neutral axis positions of the cylinder and ring.

\section{The governing equations and boundary conditions}

In this study, the equations of motion are derived using Hamilton's principle, the modified couple stress, and first-order shear deformation theories. For this purpose, one must substitute the components of the displacement field into the definition of classical and non-classical strains and, then, substitute these components into strain potential energy and kinetic energy equations. 
Components of strain field are obtained as follows [30]:

$$
\begin{aligned}
\varepsilon_{r r} & =\frac{\partial u_{r}}{\partial r}, \\
\varepsilon_{\theta \theta} & =\frac{1}{r}\left[\frac{\partial u_{\theta}}{\partial \theta}+u_{r}\right], \\
\varepsilon_{z z} & =\frac{\partial u_{z}}{\partial z}, \\
\varepsilon_{r \theta} & =\frac{1}{2}\left[\frac{1}{r} \frac{\partial u_{r}}{\partial \theta}+\frac{\partial u_{\theta}}{\partial r}-\frac{u_{\theta}}{r}\right], \\
\varepsilon_{r z} & =\frac{1}{2}\left[\frac{\partial u_{r}}{\partial z}+\frac{\partial u_{z}}{\partial r}\right], \\
\varepsilon_{\theta z} & =\frac{1}{2}\left[\frac{\partial u_{\theta}}{\partial z}+\frac{1}{r} \frac{\partial u_{z}}{\partial \theta}\right] .
\end{aligned}
$$

By substituting displacement field relations (Eqs. (7) and (8)) into Eq. (11), strain fields are respectively obtained for cylinder and ring.

Non-zero components of symmetric rotation gradient tensor are obtained as follows [30]:

$$
\begin{aligned}
\chi_{r r}= & \frac{1}{2}\left[-\frac{\partial^{2} u_{\theta}}{\partial r \partial z}+\frac{1}{r} \frac{\partial^{2} u_{z}}{\partial r \partial \theta}-\frac{1}{r^{2}} \frac{\partial u_{z}}{\partial \theta}\right], \\
\chi_{\theta \theta}= & \frac{1}{2}\left[\frac{\partial^{2} u_{r}}{\partial \theta \partial z}-\frac{1}{r} \frac{\partial^{2} u_{z}}{\partial r \partial \theta}-\frac{1}{r} \frac{\partial u_{\theta}}{\partial z}+\frac{1}{r^{2}} \frac{\partial u_{z}}{\partial \theta}\right], \\
\chi_{z z}= & \frac{1}{2}\left[-\frac{1}{r} \frac{\partial^{2} u_{r}}{\partial \theta \partial z}+\frac{\partial^{2} u_{\theta}}{\partial r \partial z}+\frac{1}{r} \frac{\partial u_{\theta}}{\partial z}\right], \\
\chi_{r \theta}= & \frac{1}{4}\left[\frac{\partial^{2} u_{r}}{\partial r \partial z}-\frac{1}{r} \frac{\partial^{2} u_{\theta}}{\partial \theta \partial z}-\frac{\partial^{2} u_{z}}{\partial r^{2}}-\frac{1}{r^{2}} \frac{\partial^{2} u_{z}}{\partial \theta^{2}}\right. \\
& \left.-\frac{1}{r} \frac{\partial u_{r}}{\partial z}+\frac{1}{r} \frac{\partial u_{z}}{\partial r}\right], \\
& \left.+\frac{1}{r^{2}} \frac{\partial u_{\theta}}{\partial \theta}\right] \cdot \\
\chi_{r z}= & \frac{1}{4}\left[-\frac{1}{r} \frac{\partial^{2} u_{r}}{\partial r \partial \theta}+\frac{\partial^{2} u_{\theta}}{\partial r^{2}}-\frac{\partial^{2} u_{\theta}}{\partial z^{2}}+\frac{1}{r} \frac{\partial^{2} u_{z}}{\partial \theta \partial z}\right. \\
& \left.+\frac{1}{r^{2}} \frac{\partial u_{r}}{\partial \theta}+\frac{1}{r} \frac{\partial u_{\theta}}{\partial r}-\frac{u_{\theta}}{r}\right], \\
\chi_{\theta z} & {\left[-\frac{1}{r^{2}} \frac{\partial^{2} u_{r}}{\partial \theta^{2}}+\frac{\partial^{2} u_{r}}{\partial z^{2}}+\frac{1}{r} \frac{\partial^{2} u_{\theta}}{\partial r \partial \theta}-\frac{\partial^{2} u_{z}}{\partial r \partial z}\right.}
\end{aligned}
$$

By substituting displacement field relations (Eqs. (7) and (8)) into Eq. (12), components of symmetric rotation gradient tensor will be respectively obtained for cylinder and ring.

To measure strain energy, equations Eqs. (11) and (12) for cylinder and ring are substituted into Eq. (3); thus, the strain energy has been computed for cylinder and ring as follows:

$$
\begin{aligned}
& U_{\text {cylinder }}=\int_{0}^{2 \pi} \int_{0}^{L}\left[N_{z z} \frac{\partial \delta U_{0}}{\partial z}\right. \\
& +\left(N_{z \theta}+\frac{Y_{\theta \theta}}{2 R^{2}}-\frac{Y_{r r}}{2 R^{2}}\right) \frac{\partial \delta U_{0}}{\partial \theta} \\
& -\frac{Y_{r \theta}}{2 R^{2}} \frac{\partial^{2}\left(\delta U_{0}\right)}{\partial \theta^{2}}+\frac{Y_{r z}}{2 R^{2}} \frac{\partial^{2}\left(\delta U_{0}\right)}{\partial \theta \partial z} \\
& +\left(\frac{N_{\theta \theta}}{R}-\frac{Y_{\theta z}}{2 R^{2}}\right) \frac{\partial \delta V_{0}}{\partial \theta} \\
& +\left(N_{\theta z}-\frac{Y_{\theta \theta}}{2 R}+\frac{Y_{z z}}{2 R}\right) \frac{\partial \delta V_{0}}{\partial z} \\
& -\frac{Y_{r z}}{2} \frac{\partial^{2}\left(\delta V_{0}\right)}{\partial z^{2}}-\frac{Y_{r \theta}}{2 R} \frac{\partial^{2}\left(\delta V_{0}\right)}{\partial \theta \partial z} \\
& -\left(\frac{Q_{r \theta}}{R}+\frac{Y_{r z}}{2 R^{2}}\right) \delta V_{0}+\frac{N_{\theta \theta}}{R} \delta W_{0} \\
& +\left(\frac{Q_{r \theta}}{R}+\frac{Y_{r z}}{2 R^{2}}\right) \frac{\partial \delta W_{0}}{\partial \theta} \\
& +\left(Q_{r z}-\frac{Y_{r \theta}}{2 R}\right) \frac{\partial \delta W_{0}}{\partial z}+\frac{Y_{\theta z}}{2} \frac{\partial^{2}\left(\delta W_{0}\right)}{\partial z^{2}} \\
& -\frac{Y_{\theta z}}{2 R^{2}} \frac{\partial^{2}\left(\delta W_{0}\right)}{\partial \theta^{2}}+\left(\frac{Y_{\theta \theta}}{2}-\frac{Y_{z z}}{2 R}\right) \frac{\partial^{2}\left(\delta W_{0}\right)}{\partial \theta \partial z} \\
& +Q_{r z} \delta \psi_{z}+\left(\frac{M_{z \theta}}{R}+\frac{Y_{r r}}{2 R}-\frac{Y_{\theta \theta}}{2 R}\right) \frac{\partial \delta \psi_{z}}{\partial \theta} \\
& +\left(M_{z \theta}-\frac{Y_{\theta z}}{2}\right) \frac{\partial \delta \psi_{z}}{\partial z}-\frac{T_{r \theta}}{2 R} \frac{\partial^{2}\left(\delta \psi_{z}\right)}{\partial \theta^{2}} \\
& +\frac{T_{r z}}{2 R} \frac{\partial^{2}\left(\delta \psi_{z}\right)}{\partial \theta \partial z}+\left(Q_{r \theta}+\frac{Y_{r z}}{2 R}\right) \delta \psi_{\theta} \\
& +\left(\frac{M_{\theta \theta}}{R}+\frac{Y_{\theta z}}{2 R}\right) \frac{\partial \delta \psi_{\theta}}{\partial \theta} \\
& +\left(M_{z \theta}+\frac{Y_{z z}}{2}-\frac{Y_{r r}}{2}-\frac{T_{\theta \theta}}{2 R}\right) \frac{\partial \delta \psi_{\theta}}{\partial z} \\
& \left.-\frac{T_{r z}}{2} \frac{\partial^{2}\left(\delta \psi_{\theta}\right)}{\partial z^{2}}-\frac{T_{r \theta}}{2 R} \frac{\partial^{2}\left(\delta \psi_{\theta}\right)}{\partial \theta \partial z}\right] R d z d \theta
\end{aligned}
$$




$$
\begin{aligned}
U_{\text {ring }}= & \int_{0}^{2 \pi} \int_{0}^{L}\left[\left(N_{z \theta}^{\prime}+\frac{Y_{\theta \theta}^{\prime}}{2 R^{\prime 2}}-\frac{Y_{r r}^{\prime}}{2 R^{\prime 2}}\right) \frac{\partial U_{0}}{\partial \theta}\right. \\
& -\frac{Y_{r \theta}^{\prime}}{2 R^{\prime 2}} \frac{\partial^{2}\left(U_{0}\right)}{\partial \theta^{2}}+\left(\frac{N_{\theta \theta}^{\prime}}{R^{\prime}}-\frac{Y_{\theta z}^{\prime}}{2 R^{\prime 2}}\right) \frac{\partial V_{0}}{\partial \theta} \\
& -\left(\frac{Q_{r \theta}^{\prime}}{R^{\prime}}+\frac{Y_{r z}^{\prime}}{2 R^{\prime 2}}\right) V_{0}+\frac{N_{\theta \theta}^{\prime}}{R^{\prime}} W_{0} \\
& +\left(\frac{Q_{r \theta}^{\prime}}{R^{\prime}}+\frac{Y_{r z}^{\prime}}{2 R^{\prime 2}}\right) \frac{\partial W_{0}}{\partial \theta}-\frac{Y_{\theta z}^{\prime}}{2 R^{\prime 2}} \frac{\partial^{2}\left(W_{0}\right)}{\partial \theta^{2}} \\
& +Q_{r z}^{\prime} \psi_{z}+\left(\frac{M_{z \theta}^{\prime}}{R^{\prime}}+\frac{Y_{r r}^{\prime}}{2 R^{\prime}}-\frac{Y_{\theta \theta}^{\prime}}{2 R^{\prime}}\right) \frac{\partial \psi_{z}}{\partial \theta} \\
& -\frac{T_{r \theta}^{\prime}}{2 R^{\prime}} \frac{\partial^{2}\left(\psi_{z}\right)}{\partial \theta^{2}}+\left(Q_{r \theta}^{\prime}+\frac{Y_{r z}^{\prime}}{2 R^{\prime}}\right) \psi_{\theta} \\
& \left.+\left(\frac{M_{\theta \theta}^{\prime}}{R^{\prime}}+\frac{Y_{\theta}^{\prime} z}{2 R^{\prime}}\right) \frac{\partial \psi_{\theta}}{\partial \theta}\right] R d z d \theta,
\end{aligned}
$$

where classical and non-classical forces and momentum for cylinder and ring are defined as follows:

$$
\begin{gathered}
\left(N_{z z}, N_{\theta \theta}, N_{z \theta}\right)=\int_{-r_{c}}^{h-r_{c}}\left(\sigma_{z z}, \sigma_{\theta \theta}, \sigma_{z \theta}\right) d r \\
\left(M_{z z}, M_{\theta \theta}, M_{z \theta}\right)=\int_{-r_{c}}^{h-r_{c}}\left(\sigma_{z z}, \sigma_{\theta \theta}, \sigma_{z \theta}\right) r d r \\
\left(Q_{z r}, Q_{r \theta}\right)=\int_{-r_{c}}^{h-r_{c}}\left(\sigma_{z r}, \sigma_{r \theta}\right) d r, \\
\left(Y_{z z}, Y_{\theta \theta}, Y_{r r}, Y_{z \theta}, Y_{z r}, Y_{r \theta}\right) \\
\int_{-r_{c}}^{h-r_{c}}\left(m_{z z}, m_{\theta \theta}, m_{r r}, m_{z \theta}, m_{z r}, m_{r \theta}\right) d r \\
\left(T_{z z}, T_{\theta \theta}, T_{r r}, T_{z \theta}, T_{z r}, T_{r \theta}\right) \\
\left.M_{z-r_{c}}^{\prime}, M_{\theta \theta}^{\prime}, N_{z \theta}^{\prime}\right)=\int_{-r_{r c}}^{\left.M_{z \theta}^{\prime}\right)}\left(\sigma_{z z}, \sigma_{\theta \theta}, \sigma_{z \theta}\right) d r \\
=\int_{-r_{c}}^{h_{r}-r_{r c}}\left(m_{z z}, m_{\theta \theta}, m_{r r}, m_{z \theta}, m_{z r}, m_{r \theta}\right) r d r
\end{gathered}
$$

$$
\begin{aligned}
& \left(Q_{z r}^{\prime}, Q_{r \theta}^{\prime}\right)=\int_{-r_{r c}}^{h_{r}-r_{r c}}\left(\sigma_{z r}, \sigma_{r \theta}\right) d r, \\
& \left(Y_{z z}^{\prime}, Y_{\theta \theta}^{\prime}, Y_{r r}^{\prime}, Y_{z \theta}^{\prime}, Y_{z r}^{\prime}, Y_{r \theta}^{\prime}\right) \\
& =\int_{-r_{r c}}^{h_{r}-r_{r c}}\left(m_{z z}, m_{\theta \theta}, m_{r r}, m_{z \theta}, m_{z r}, m_{r \theta}\right) d r \\
& \left(T_{z z}^{\prime}, T_{\theta \theta}^{\prime}, T_{r r}^{\prime}, T_{z \theta}^{\prime}, T_{z r}^{\prime}, T_{r \theta}^{\prime}\right) \\
& =\int_{-r_{r c}-r_{r c}}\left(m_{z z}, m_{\theta \theta}, m_{r r}, m_{z \theta}, m_{z r}, m_{r \theta}\right) r d r .
\end{aligned}
$$

Variation in kinetic energy of the cylindrical shell is expressed by considering displacement field for cylinder and ring as follows:

$$
\begin{aligned}
T_{\text {cylinder }}= & \frac{1}{2} \int_{\Omega} \rho(\hat{r})\left[\left(\frac{\partial U_{0}}{\partial t}+r \frac{\partial \psi_{z}}{\partial t}\right)^{2}\right. \\
& \left.+\left(\frac{\partial V_{0}}{\partial t}+r \frac{\partial \psi_{\theta}}{\partial t}\right)^{2}+\left(\frac{\partial W_{0}}{\partial t}\right)^{2}\right] R d r d \theta d z \\
T_{\text {ring }}= & \frac{1}{2} \int_{\Omega} \rho\left(\hat{r}^{\prime}\right)\left[\left(\frac{\partial U_{0}}{\partial t}+r^{\prime} \frac{\partial \psi_{z}}{\partial t}\right)^{2}\right. \\
& \left.+\left(\frac{\partial V_{0}}{\partial t}+r^{\prime} \frac{\partial \psi_{\theta}}{\partial t}\right)^{2}+\left(\frac{\partial W_{0}}{\partial t}\right)^{2}\right] R^{\prime} d r^{\prime} d \theta d z
\end{aligned}
$$

By considering strain and kinetic energies of the cylinder and ring, Lagrangian function can be expressed as follows:

$$
F=U-T+\sum_{i=1}^{N_{s}} U_{r_{i}}-T_{r_{i}}
$$

where $N_{s}$ is the number of stiffeners.

By using variational method, the governing equations of motion and boundary conditions have been derived, as shown in Appendix A.

\section{Solution}

In this section, simply-supported boundary condition exerted on both ends of the structure and free vibration of FGM stiffened micro cylinder has been investigated by Navier procedure.

For simply-supported condition, due to the variability of $\theta$, boundary conditions in Eqs. (A.15) to (A.22) are satisfied, and only boundary conditions 
with $z=$ constant must be set up. Due to the freedom of the two ends of the cylindrical shell from bending moment and higher order stresses, essential and natural boundary conditions in the classical and non-classical cases are as follows:

$$
\begin{aligned}
& \left.V\right|_{z=0, L}=0, \\
& \left.W\right|_{z=0, L}=0, \\
& \left.\delta \psi_{\theta}\right|_{z=0, L}=0 \\
& {\left[D_{10} \frac{\partial U}{\partial z}-\frac{D_{50} l^{2}}{4 R^{2}} \frac{\partial^{3} U}{\partial z \partial \theta^{2}}+\frac{D_{50} l^{2}}{4 R} \frac{\partial^{3} V}{\partial z^{2} \partial \theta}\right.} \\
& +\frac{1}{R}\left(D_{30}+\frac{D_{50} l^{2}}{4 R^{2}}\right) \frac{\partial V}{\partial \theta}+\frac{D_{30}}{R} W \\
& -\frac{D_{50} l^{2}}{4 R^{3}} \frac{\partial^{2} W}{\partial \theta^{2}}+\frac{1}{R}\left(D_{31}-\frac{D_{50} l^{2}}{4 R}\right) \frac{\partial \psi_{\theta}}{\partial \theta} \\
& +D_{11} \frac{\partial \psi_{z}}{\partial z}+\frac{D_{51} l^{2}}{4 R} \frac{\partial^{3} \psi_{\theta}}{\partial z^{2} \partial \theta}-\frac{D_{51} l^{2}}{4 R^{2}} \frac{\partial^{3} \psi_{z}}{\partial z \partial \theta^{2}} \\
& +\sum_{i=1}^{N_{s}} \frac{1}{R^{\prime}}\left(R_{30}+\frac{R_{50} l^{2}}{4 R^{\prime 2}}\right) \frac{\partial V}{\partial \theta} \delta\left(z-z_{i}\right) \\
& +\frac{R_{30}}{R^{\prime}} W \delta\left(z-z_{i}\right)-\frac{R_{50} l^{2}}{4 R^{\prime 3}} \frac{\partial^{2} W}{\partial \theta^{2}} \delta\left(z-z_{i}\right) \\
& \left.+\frac{1}{R^{\prime}}\left(R_{31}-\frac{R_{50} l^{2}}{4 R^{\prime}}\right) \frac{\partial \psi_{\theta}}{\partial \theta} \delta\left(z-z_{i}\right)\right]\left.d \theta\right|_{z=0, L}=0
\end{aligned}
$$

$$
\begin{gathered}
{\left[\frac{D_{50} l^{2}}{4} \frac{\partial^{2} V}{\partial z^{2}}+\frac{D_{50} l^{2}}{4 R^{2}} V-\frac{D_{50} l^{2}}{4 R} \frac{\partial^{2} U}{\partial z \partial \theta}-\frac{D_{50} l^{2}}{4 R^{2}} \frac{\partial W}{\partial \theta}\right.} \\
-\frac{D_{50} l^{2}}{4 R} \psi_{\theta}+\frac{D_{51} l^{2}}{4} \frac{\partial^{2} \psi_{\theta}}{\partial z^{2}}-\frac{D_{51} l^{2}}{4 R} \frac{\partial^{2} \psi_{z}}{\partial z \partial \theta} \\
+\sum_{i=1}^{N_{s}} \frac{R_{50} l^{2}}{4 R^{\prime 2}} V \delta\left(z-z_{i}\right)-\frac{R_{50} l^{2}}{4 R^{\prime}} \psi_{\theta} \delta\left(z-z_{i}\right) \\
\left.-\frac{R_{50} l^{2}}{4 R^{\prime 2}} \frac{\partial W}{\partial \theta} \delta\left(z-z_{i}\right)\right]\left.d \theta\right|_{z=0, L}=0,
\end{gathered}
$$

$$
\begin{array}{r}
{\left[\frac{D_{50} l^{2}}{4} \frac{\partial^{2} W}{\partial z^{2}}-\frac{D_{50} l^{2}}{4 R^{2}} \frac{\partial^{2} W}{\partial \theta^{2}}+\frac{D_{50} l^{2}}{4 R^{2}} \frac{\partial V}{\partial \theta}\right.} \\
+\frac{D_{50} l^{2}}{4 R} \frac{\partial \psi_{\theta}}{\partial \theta}-\frac{D_{50} l^{2}}{4} \frac{\partial \psi_{z}}{\partial z}+\sum_{i=1}^{N_{s}}
\end{array}
$$

$$
\begin{aligned}
& -\frac{R_{50} l^{2}}{4 R^{\prime 2}} \frac{\partial^{2} W}{\partial \theta^{2}} \delta\left(z-z_{i}\right)+\frac{R_{50} l^{2}}{4 R^{\prime 2}} \frac{\partial V}{\partial \theta} \delta\left(z-z_{i}\right) \\
& \left.+\frac{R_{50} l^{2}}{4 R^{\prime}} \frac{\partial \psi_{\theta}}{\partial \theta} \delta\left(z-z_{i}\right)\right]\left.d \theta\right|_{z=0, L}=0 \\
& {\left[-\frac{D_{50} l^{2}}{4} \frac{\partial^{2} W}{\partial z^{2}}+\frac{1}{R^{2}}\left(\frac{D_{50} l^{2}}{4}-\frac{D_{51} l^{2}}{4 R}\right) \frac{\partial^{2} W}{\partial \theta^{2}}\right.} \\
& +\frac{1}{R}\left(D_{32}-\frac{D_{50} l^{2}}{4}-\frac{D_{51} l^{2}}{4 R}\right) \frac{\partial \psi_{\theta}}{\partial \theta} \\
& +\left(D_{12}+\frac{D_{50} l^{2}}{4}\right) \frac{\partial \psi_{z}}{\partial z} \\
& +\frac{1}{R}\left(D_{31}-\frac{D_{50} l^{2}}{4 R}+\frac{D_{51} l^{2}}{4 R^{2}}\right) \frac{\partial V}{\partial \theta} \\
& -\frac{D_{52} l^{2}}{4 R^{2}} \frac{\partial^{3} \psi_{z}}{\partial z \partial \theta^{2}}+\frac{D_{52} l^{2}}{4 R} \frac{\partial^{3} \psi_{\theta}}{\partial z^{2} \partial \theta} \\
& -\frac{D_{51} l^{2}}{4 R^{2}} \frac{\partial^{3} U}{\partial z \partial \theta^{2}}+\frac{D_{51} l^{2}}{4 R} \frac{\partial^{3} V}{\partial z^{2} \partial \theta} \\
& +\sum_{i=1}^{N_{s}} \frac{1}{R^{\prime}}\left(R_{32}-\frac{R_{50} l^{2}}{4}-\frac{R_{51} l^{2}}{4 R^{\prime}}\right) \frac{\partial \psi_{\theta}}{\partial \theta} \delta\left(z-z_{i}\right) \\
& +\frac{1}{R^{\prime}}\left(R_{31}-\frac{R_{50} l^{2}}{4 R^{\prime}}+\frac{R_{51} l^{2}}{4 R^{\prime 2}}\right) \frac{\partial V}{\partial \theta} \delta\left(z-z_{i}\right) \\
& \left.+\frac{1}{R^{\prime 2}}\left(\frac{R_{50} l^{2}}{4}-\frac{R_{51} l^{2}}{4 R^{\prime}}\right) \frac{\partial^{2} W}{\partial \theta^{2}} \delta\left(z-z_{i}\right)\right]\left.d \theta\right|_{z=0, L} \\
& =0 \text {, } \\
& {\left[-\frac{D_{52} l^{2}}{4 R} \frac{\partial^{2} \psi_{z}}{\partial z \partial \theta}+\frac{D_{52} l^{2}}{4} \frac{\partial^{2} \psi_{\theta}}{\partial z^{2}}+\frac{D_{51} l^{2}}{4 R^{2}} V\right.} \\
& -\frac{D_{51} l^{2}}{4 R} \frac{\partial^{2} U}{\partial z \partial \theta}+\frac{D_{51} l^{2}}{4} \frac{\partial^{2} V}{\partial z^{2}}-\frac{D_{51} l^{2}}{4 R^{2}} \frac{\partial W}{\partial \theta} \\
& -\frac{D_{51} l^{2}}{4 R} \psi_{\theta}+\sum_{i=1}^{N_{s}} \frac{R_{51} l^{2}}{4{R^{\prime}}^{2}} V \delta\left(z-z_{i}\right) \\
& -\frac{R_{51} l^{2}}{4 R^{\prime 2}} \frac{\partial W}{\partial \theta} \delta\left(z-z_{i}\right) \\
& \left.-\frac{R_{51} l^{2}}{4 R^{\prime}} \psi_{\theta} \delta\left(z-z_{i}\right)\right]\left.d \theta\right|_{z=0, L}=0
\end{aligned}
$$

For simply-supported condition at the both ends of the structure and using Navier method, independent variables $U, V, W, \psi_{\theta}(\theta, t)$, and $\psi_{z}(\theta, t)$ are assumed 
as binary series of trigonometric functions for ring and cylinder as follows:

$$
\begin{aligned}
& U(z, \theta, t)=\sum_{n} \sum_{m} U_{m n}(t) \cos \left(\frac{m \pi}{L} z\right) \cos (n \theta), \\
& V(z, \theta, t)=\sum_{n} \sum_{m} V_{m n}(t) \sin \left(\frac{m \pi}{L} z\right) \sin (n \theta), \\
& W(z, \theta, t)=\sum_{n} \sum_{m} W_{m n}(t) \sin \left(\frac{m \pi}{L} z\right) \cos (n \theta), \\
& \psi_{\theta}(z, \theta, t)=\sum_{n} \sum_{m} \psi_{\theta m n}(t) \sin \left(\frac{m \pi}{L} z\right) \sin (n \theta), \\
& \psi_{z}(z, \theta, t)=\sum_{n} \sum_{m} \psi_{z m n}(t) \cos \left(\frac{m \pi}{L} z\right) \cos (n \theta), \\
& U(\theta, t)=\sum_{n} \sum_{m} U_{m n}(t) \cos (n \theta), \\
& \left.\psi_{z}(\theta, t)=\sum_{n} \sum_{m} \psi_{z m n}(t) \cos (n \theta), t\right)=\sum_{n} \sum_{m} V_{m n}(t) \sin (n \theta), \\
& W(\theta, t)=\sum_{n} \sum_{m} W_{m n}(t) \cos (n \theta), \\
& \psi_{\theta}(\theta, t)=\sum_{m} \psi_{\theta m n}(t) \sin (n \theta),
\end{aligned}
$$

where $m$ and $n$ are the axial and circumferential wave numbers. By substituting Eqs. (28) and (29) into equations of motion (Eqs. (A.2) through (A.6)), equations can be written in the matrix form as follows:

$$
\left[[K]+\sum_{i=1}^{N_{s}}\left[K r_{i}\right]\right]\{C\}+\left[[M]+\sum_{i=1}^{N_{s}}\left[M r_{i}\right]\right]\{\ddot{C}\}=\{0\}
$$

where $[K]$ and $[M]$ are the stiffness and mass matrix of the cylinder, while $[K r]$ and $[M r]$ are the stiffness and mass matrix of the ring stiffener. In addition, $\{C\}$ is defined as follows:

$$
\{C\}=\left\{C_{0}\right\} e^{i \omega t} .
$$

By substituting Eq. (31) into Eq. (30), the equation can be rewritten as follows:

$$
\left[[K]+\sum_{i=1}^{N_{s}}\left[K r_{i}\right]-\omega^{2}\left([M]+\sum_{i=1}^{N_{s}}\left[M r_{i}\right]\right)\right]\left\{C_{0}\right\}=\{0\},
$$

where $\omega$ is the natural frequency, and $\left\{C_{0}\right\}=$ $\left\{U_{m n}, V_{m n}, W_{m n}, \psi_{z_{m n}}, \psi_{\theta_{m n}}\right\}^{T}$ is displacement amplitude vector. To find natural frequencies, one must set the determinant of coefficient equal to zero.
Table 1. Material properties of FGM.

\begin{tabular}{cccc}
\hline & $\boldsymbol{E}(\mathbf{G P a})$ & $\boldsymbol{\rho}\left(\mathbf{k g} / \mathbf{m}^{\mathbf{3}}\right)$ & $\boldsymbol{\nu}$ \\
\hline $\mathrm{Al}$ & 70 & 2702 & 0.3 \\
$\mathrm{C}$ & 427 & 3100 & 0.17 \\
\hline
\end{tabular}

\section{Results}

In this section, it is considered that the FGM micro cylinder is made by ceramics in its outer layer and aluminum in its inner layer, whose mechanical properties of these materials are given in Table 1 in which $\mathrm{C}$ depicts ceramics ( $\mathrm{SiC}$ ). Results are obtained for two classical and non-classical theories; by assuming $l=$ zero in the equations of motion, the governing equations obtained by the modified couple stress theory reduce to classical continuum theory.

Effects of different parameters, such as thickness, length, dimensionless length scale parameter, circumferential wave number, stiffener and distribution of FGM properties, on the natural frequencies are investigated for the simply-supported FGM micro cylinder. Dimensionless natural frequency can be $\Omega=\omega R \sqrt{\rho / E}$.

\subsection{Effects of dimensionless length scale parameter on natural frequency}

Effects of dimensionless length scale parameter and gradient index on the natural frequency are illustrated in Figure 2 for different FGM distribution powers $(N)$ of $l=R / 1000$. As illustrated in Figures 2, 3 and 5, a decrease in dimensionless length scale parameter, $h / l$, leads to the stiffening of shell and an increase in natural frequency in the entire gradient index $(N)$.

\subsection{Effects of using one stiffener on the dimensionless natural frequency}

In this section, one ring stiffener placed in $z=L / 3$ from one end of the cylinder is assumed. Ring is also made of FGM just as the cylinder and distribution power is assumed equal to 1 for this case. Figure 3 shows the effect of using one stiffener on the dimensionless natural frequency rather than a normal cylinder. As illustrated in Figure 3, using one stiffener leads to an increase in the stiffness of the structure; so, the natural frequency increases as expected.

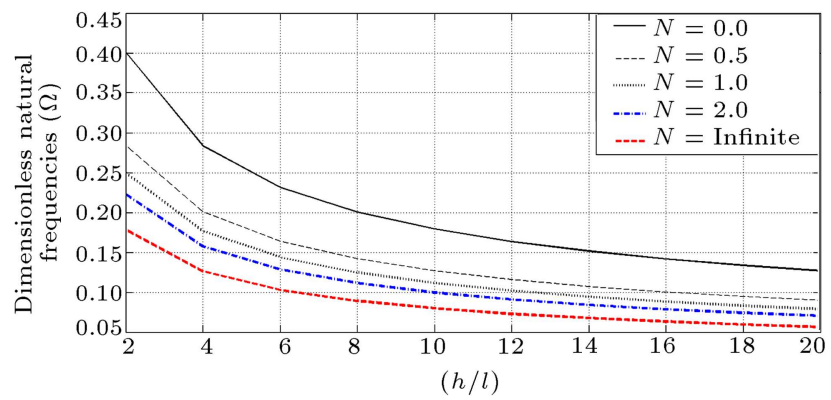

Figure 2. Effect of dimensionless length scale parameter on dimensionless natural frequencies. 


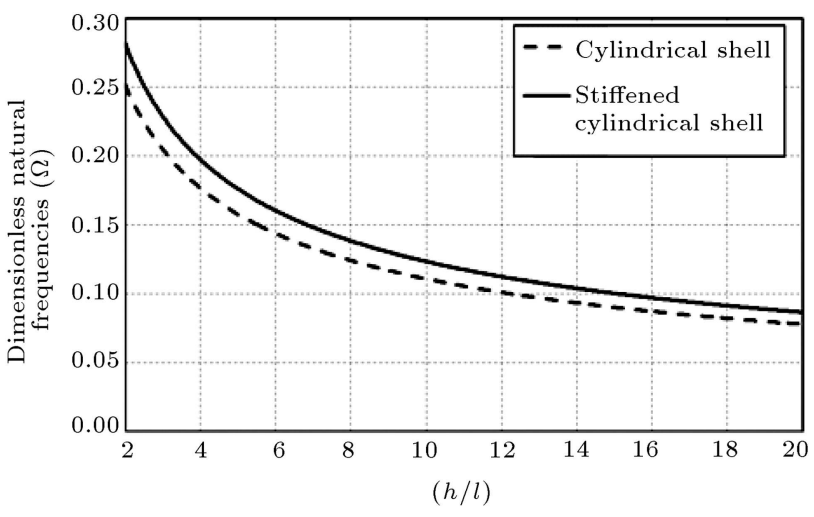

Figure 3. Effect of using one ring on the natural frequency $(n=1)$.

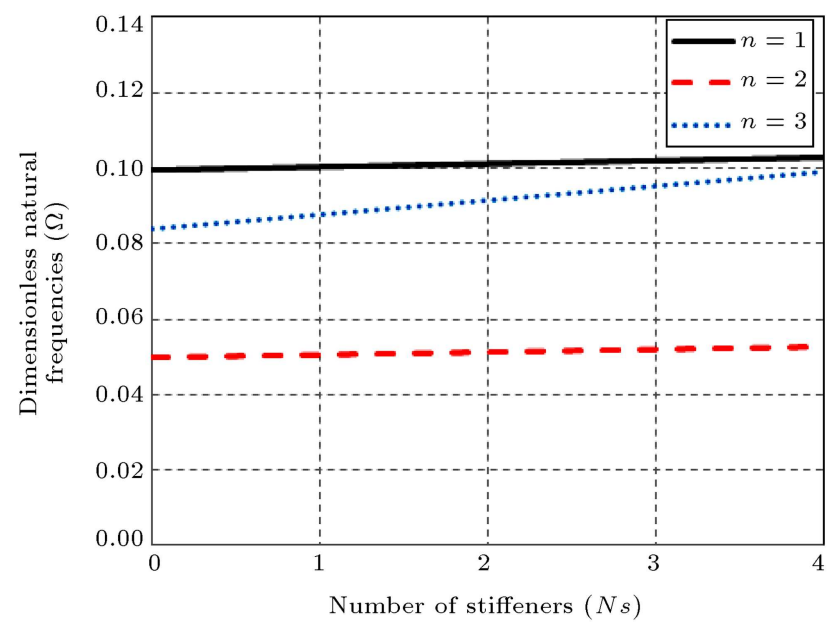

Figure 4. Dimensionless natural frequencies $(L / R=10)$.

Table 2. Geometrical properties of cylinder and ring.

\begin{tabular}{lc}
\hline \multicolumn{1}{c}{ Characteristics } & Value \\
\hline Thickness to radius ratio $(h / R)$ & 0.01 \\
Ring height to radius ratio $(h r / R)$ & 0.02 \\
Stiffening type & External \\
\hline
\end{tabular}

Figure 4 shows the effects of numerous stiffeners from 1 to 4 numbers on $L / R=10$, considering the distribution power equal to 1 and axial wave number $m=1$. The geometrical properties of ring and cylindrical shell are shown in Table 2.

\subsection{Effects of dimensionless length parameter on natural frequency}

For classical and non-classical theories, effects of dimensionless length parameter, $L / R$, and dimensionless length scale parameter, $h / l$, are investigated simultaneously and shown in Figures 5 and 6 . As shown, the decrease in the length parameter leads to a reduction of instability in the micro cylinder and, therefore, a reduction in deformation; hence, the natural frequency increases. The increase in natural frequency due to the decrease in length parameter based on the modified

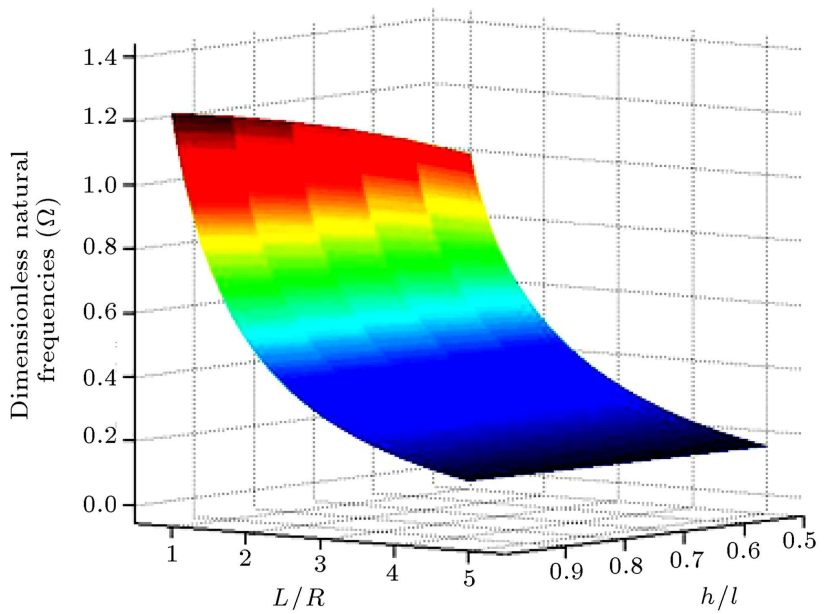

Figure 5. Effects of $L / R$ and $h / l$ on the dimensionless natural frequencies based on the classical theory.

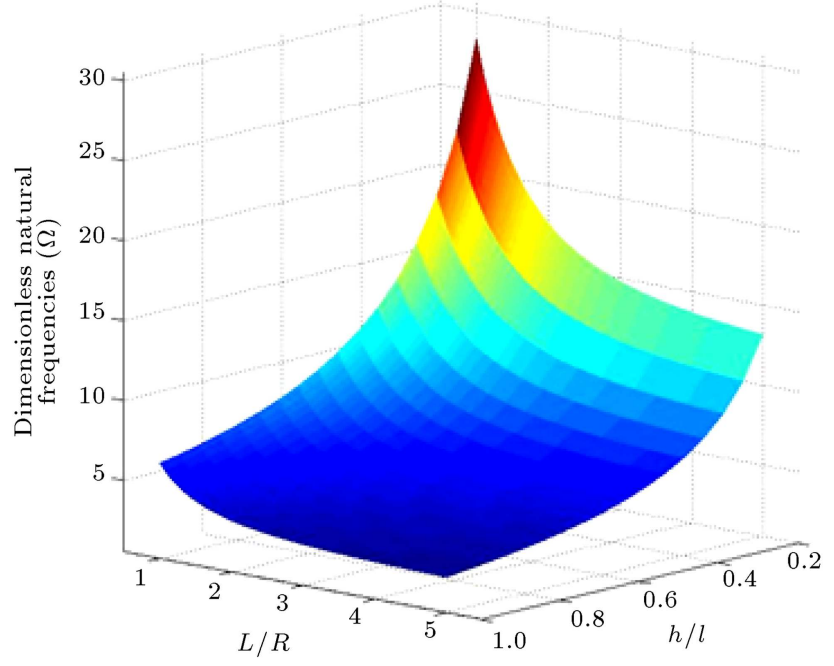

Figure 6. Effects of $L / R$ and $h / l$ on the dimensionless natural frequencies based on the modified couple stress theory.

couple stress theory has an increasing effect on the values of natural frequency in the higher values of the length scale parameter. In contrast to the modified couple stress theory, based on the classical continuum theory, variation in the dimensionless length scale parameter has no impact on the values of natural frequency in different values of dimensionless length parameter.

\subsection{Effects of the length of the cylinder on the natural frequencies}

Figure 7 directly indicates the effects of the increase in the length of the cylinder on the natural frequencies of the structure for different FGM distribution powers $(N)$. As illustrated in Figure 7, an increase in length of the unstable cylinder of the structure leads to a decrease in the natural frequency. 


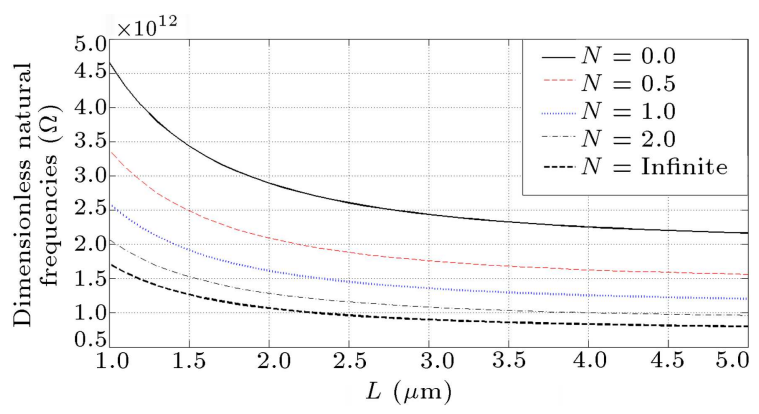

Figure 7. Effect of the length of the cylinder on the dimensionless natural frequencies for different $N(n=1)$.

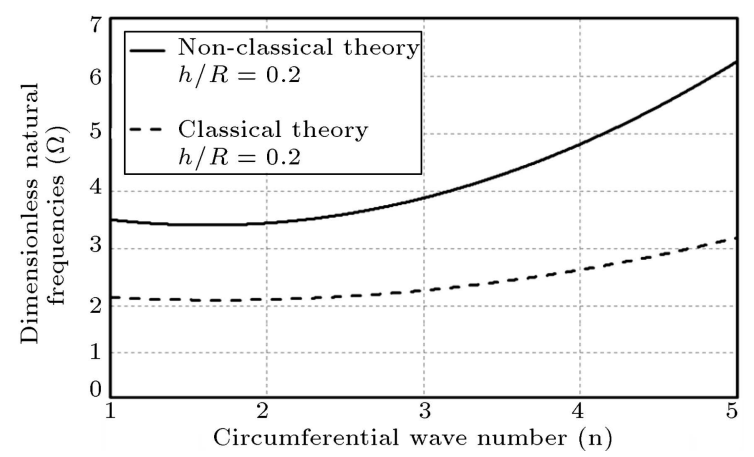

Figure 8. Effects of different circumferential wave number on the natural frequency for classical and non-classical theories.

\subsection{Effect of the circumferential wave number (n) on the natural frequencies}

Figure 8 illustrates the effects of circumferential and axial wavenumbers as well as those of the dimensionless cylindrical shell thickness parameter on dimensionless natural frequency for both classical and non-classical theories.

As is clear, an increase in circumferential wave number leads to an increase in natural frequency; due to the increase in shell thickness, the increase of natural frequency is intensified by the increase in circumferential wave number.

In Figure 9, a comparison is made between results of the current study and those of the Ref [30], showing

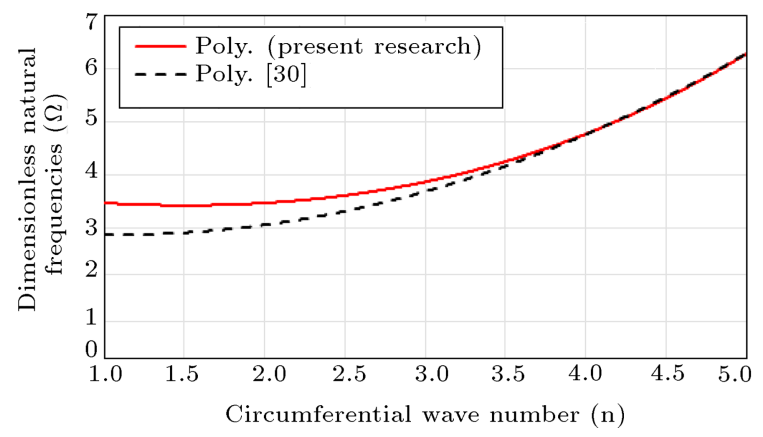

Figure 9. Comparison of the results of the present research with $[30] ; h / R=0.2$. that as the circumferential wave number increases, the results converge.

\section{Conclusion}

In this paper, free vibration of microcylinder made of functionally graded materials stiffened in Circumferential direction was investigated based on the modified couple stress and first-order shear deformation theories. Material properties of the FGM shell were assumed as continuously variable along thickness. The Modified Couple Stress Theory (MCST) was utilized to capture size effects in micro scales; moreover, the general equations and boundary conditions were derived considering first-order shear deformation theory and using Hamilton's principle. Finally, simply-supported boundary conditions were assumed as a common case, and free vibration of functionally graded stiffened microcylinder was investigated. As illustrated in this paper, the classical continuum theory is unable to capture size effects in small scales; therefore, higher-order theories, such as modified couple stress theory, must be used to investigate these effects. Size-dependent effects are significant when the thickness of the cylinder is small; as the thickness increases, size effects can be neglected.

It is shown that using ring stiffeners leads to the increase of natural frequency. Furthermore, it is depicted that for a typical $L / R=10$, as the number of ring stiffeners increases, the dimensionless natural frequency for different axial wave numbers increases, too. This event can be justified by the fact that ring stiffeners increase the stiffness of the structure and corresponding stiffness matrix; therefore, the natural frequency increases.

As shown, given different conditions and desired vibrational behavior, the application of functionally graded materials provides designers with the capability to design their preferred structures. It is concluded that different natural frequencies can be obtained using functionally graded materials with different distribution powers.

\section{Acknowledgement}

This paper is supported by INSF.

\section{References}

1. Basdekas, N.L. and Chi, M. "Response of oddly stiffened circular cylindrical shell", Journal of Sound and Vibration, 17, pp.187-206 (1971).

2. Zhou, X.P. "Vibration and stability of ring-stiffened thin-walled cylindrical shells conveying fluid", Acta Mechanica Solida Sinica, 25, pp. 168-176 (2012).

3. Hoppmann, W.H. "Some characteristics of the flexural vibrations of orthogonally stiffened cylindrical shells", 
The Journal of the Acoustical Society of America, 30(1), pp. 77-82 (1958).

4. Mikulas, M.M. and McElman, J.A., On Free Vibrations of Eccentrically Stiffened Cylindrical Shells and Flat Plates, National Aeronautics and Space Administration (1965).

5. Sankar, B.V. "An elasticity solution for functionally graded beams", Composites Science and Technology, 61(5), pp. 689-696 (2001).

6. Aydogdu, M. and Taskin, V. "Free vibration analysis of functionally graded beams with simply supported edges", Materials \& Design, 28(5), pp. 1651-1656 (2007).

7. Ying, J., Lü, C.F., and Chen, W.Q. "Two-dimensional elasticity solutions for functionally graded beams resting on elastic foundations", Composite Structures, 84(3), pp. 209-219 (2008).

8. Xiang, H.J. and Yang, J. "Free and forced vibration of a laminated FGM Timoshenko beam of variable thickness under heat conduction", Composites Part B: Engineering, 39(2), pp. 292-303 (2008).

9. Kapuria, S., Bhattacharyya, M., and Kumar, A.N. "Bending and free vibration response of layered functionally graded beams: a theoretical model and its experimental validation", Composite Structures, 82(3), pp. 390-402 (2008).

10. Prakash, T., Singha, M.K., and Ganapathi, M. "Thermal snapping of functionally graded materials plates", Materials \& Design, 30(10), pp. 4532-4536 (2009).

11. Koiter, W.T. "Couple-stresses in the theory of elasticity I and II", Proc. K Ned Akad Wet B, 67, pp. 17-44 (1969).

12. Mindlin, R.D. and Tiersten, H.F. "Effects of couplestresses in linear elasticity", Arch. Ration. Mech. Anal., 11(1), pp. 415-48 (1962).

13. Toupin, R.A. "Elastic materials with couple-stresses", Arch. Ration. Mech. Anal., 11(1), pp. 385-414 (1962).

14. Yang, F., Chong, A.C.M., Lam, D.C.C., and Tong, P. "Couple stress based strain gradient theory for elasticity", Int. J. Solids Struct., 39(10), pp. 2731-2743 (2002).

15. Zeighampour, H. and Tadi Beni, Y. "Cylindrical thinshell model based on modified strain gradient theory", International Journal of Engineering Science, 78, pp. 27-47 (2014).

16. Zhou, X., Lin, W., and Peng, Q. "Free vibration of micro-and nano-shells based on modified couple stress theory", Journal of Computational and Theoretical Nanoscience, 9(6), pp. 814-818 (2012).

17. Hosseini-Hashemi, Sh., Sharifpour, F., and Ilkhani, M.R. "On the free vibrations of size-dependent closed micro/nano-spherical shell based on the modified couple stress theory", International Journal of Mechanical Sciences, 115, pp. 501-515 (2016).

18. Park, S.K. and Gao, X.L. "Bernoulli-Euler beam model based on a modified couple stress theory", Micromech Microeng, 16(11), pp. 2355-2359 (2006).
19. Kong, S., Zhou, S., Nie, Z., and Wang, K. "The sizedependent natural frequency of Bernoulli Euler microbeams", Int. J. Eng. Sci, 46, pp. 427-437 (2008).

20. Şimşek, M. "Nonlinear static and free vibration analysis of microbeams based on the nonlinear elastic foundation using modified couple stress theory and He's variational method", Composite Structures, 112, pp. 264-272 (2014).

21. Akgöz, B. and Civalek, Ö. "Strain gradient elasticity and modified couple stress models for buckling analysis of axially loaded micro-scaled beams", International Journal of Engineering Science, 49(11), pp. 1268-1280 (2011).

22. Ghayesh, M.H., Farokhi, H., and Amabili, M. "Nonlinear dynamics of a microscale beam based on the modified couple stress theory", Composites Part B: Engineering, 50, pp. 318-324 (2013).

23. Wang, Y., Wen-Hui, L., and Ning, L. "Nonlinear free vibration of a microscale beam based on modified couple stress theory", Physica E: Low-dimensional Systems and Nanostructures, 47, pp. 80-85 (2013).

24. Jomehzadeh, E., Saidi, A.R., and Atashipour, S.R. "An analytical approach for stress analysis of functionally graded annular sector plates", Materials \& design, 30(9), pp. 3679-3685 (2009).

25. Asghari, M., Ahmadian, M.T., Kahrobaiyan, M.H., and Rahaeifard, M. "On the size-dependent behavior of functionally graded micro-beams", Materials \& Design (1980-2015), 31(5), pp. 2324-2329 (2010).

26. Asghari, M., Rahaeifard, M., Kahrobaiyan, M.H., and Ahmadian, M.T. "The modified couple stress functionally graded Timoshenko beam formulation", Materials \& Design, 32(3), pp. 1435-1443 (2011).

27. Sadeghi, H., Baghani, M., and Naghdabadi, R. "Strain gradient elasticity solution for functionally graded micro-cylinders", International Journal of Engineering Science, 50(1), pp. 22-30 (2012).

28. Sahmani, S., Ansari, R., Gholami, R., and Darvizeh, A. "Dynamic stability analysis of functionally graded higher-order shear deformable microshells based on the modified couple stress elasticity theory", Composites Part B: Engineering, 51, pp. 44-53 (2013).

29. Bedroud, M., Nazemnezhad, R., Hosseini Hashemi, S., and Valixani, M. "Buckling of FG circular/annular Mindlin nanoplates with an internal ring support using nonlocal elasticity", Applied Mathematical Modelling, 40(4), pp. 3185-3210 (2016).

30. Beni, Yaghoub Tadi, Mehralian, F. and Razavi, H. "Free vibration analysis of size-dependent shear deformable functionally graded cylindrical shell on the basis of modified couple stress theory", Composite Structures, 120, pp. 65-78 (2015).

\section{Appendix A}

By using variational method and considering the following coefficients for cylinder and ring, the governing 
equations of motion and boundary conditions have been derived:

$$
\begin{aligned}
& D_{1 i}=\int_{-r_{c}}^{h-r_{c}} \frac{E(r)}{1-\nu^{2}(r)}\left(r^{i}\right) d r, \quad i=0,1,2, \\
& D_{3 i}=\int_{-r_{c}}^{h-r_{c}} \frac{E(r) \nu(r)}{1-\nu^{2}(r)}\left(r^{i}\right) d r, \quad i=0,1,2, \\
& D_{5 i}=\int_{-r_{c}}^{h-r_{c}} \mu(r) r^{i} d r, \quad i=0,1,2, \\
& I_{1 i}=\int_{-r_{c}}^{h-r_{c}} \rho(r) r^{i} d r, \quad i=0,1,2 \\
& R_{1 i}=\int_{-r_{r c}}^{h-r_{r c}} \frac{E\left(r^{\prime}\right)}{1-\nu^{2}\left(r^{\prime}\right)}\left({r^{\prime}}^{i}\right) d r^{\prime}, \quad i=0,1,2, \\
& R_{3 i}=\int_{-r_{r c}}^{h-r_{r c}} \frac{E\left(r^{\prime}\right) \nu\left(r^{\prime}\right)}{1-\nu^{2}\left(r^{\prime}\right)}\left({r^{\prime}}^{i}\right) d r^{\prime}, \quad i=0,1,2, \\
& R_{5 i}=\int_{-r_{r c}}^{h-r_{r c}} \mu\left(r^{\prime}\right) r^{\prime i} d r^{\prime}, \quad i=0,1,2 \\
& J_{1 i}=\int_{-r_{r c}}^{h-r_{r c}} \rho\left(r^{\prime}\right) r^{\prime i} d r^{\prime}, \quad i=0,1,2,
\end{aligned}
$$

$$
\begin{aligned}
& -\frac{D_{51} l^{2}}{4 R} \frac{\partial^{4} \psi_{\theta}}{\partial z^{3} \partial \theta}-D_{11} \frac{\partial^{2} \psi_{z}}{\partial z^{2}}+\frac{D_{51} l^{2}}{4 R^{2}} \frac{\partial^{4} \psi_{z}}{\partial z^{2} \partial \theta^{2}} \\
& +\frac{\left(\frac{5 D_{50} l^{2}}{4 R}-D_{51}\right)}{R^{2}} \frac{\partial^{2} \psi_{z}}{\partial \theta^{2}}+\frac{D_{51} l^{2}}{4 R^{4}} \frac{\partial^{4} \psi_{z}}{\partial \theta^{4}} \\
& +I_{10} \frac{\partial^{2} U}{\partial t^{2}}+\sum_{i=1}^{N_{s}}-\frac{R_{50}\left(\frac{l^{2}}{R^{\prime 2}}+1\right)}{R^{\prime 2}} \frac{\partial^{2} U}{\partial \theta^{2}} \delta\left(z-z_{i}\right) \\
& +\frac{R_{50} l^{2}}{4 R^{\prime 4}} \frac{\partial^{4} U}{\partial \theta^{4}} \delta\left(z-z_{i}\right)+\frac{5 R_{50} l^{2}}{4 R^{\prime 3}} \frac{\partial^{2} \psi_{z}}{\partial \theta^{2}} \delta\left(z-z_{i}\right) \\
& +\frac{R_{51} l^{2}}{4 R^{\prime 4}} \frac{\partial^{4} \psi_{z}}{\partial \theta^{4}} \delta\left(z-z_{i}\right)+J_{10} \frac{\partial^{2} U}{\partial t^{2}} \delta\left(z-z_{i}\right),
\end{aligned}
$$

$$
\begin{aligned}
& \delta V: \frac{D_{50} l^{2}}{4} \frac{\partial^{4} V}{\partial z^{4}}-D_{50}\left(\frac{l^{2}}{2 R^{2}}+1\right) \frac{\partial^{2} V}{\partial z^{2}} \\
& -\frac{1}{R^{2}}\left(D_{10}+\frac{D_{50} l^{2}}{4 R^{2}}\right) \frac{\partial^{2} V}{\partial \theta^{2}} \\
& +\frac{D_{50} l^{2}}{4 R^{2}} \frac{\partial^{4} V}{\partial z^{2} \partial \theta^{2}}+\frac{D_{50}}{R^{2}}\left(\frac{l^{2}}{4 R^{2}}+1\right) V \\
& -\frac{D_{50} l^{2}}{4 R} \frac{\partial^{4} U}{\partial z^{3} \partial \theta}-\frac{D_{50} l^{2}}{4 R^{3}} \frac{\partial^{4} U}{\partial z \partial \theta^{3}} \\
& +\frac{1}{R}\left(\frac{D_{50} l^{2}}{4 R^{2}}-D_{30}-D_{50}\right) \frac{\partial^{2} U}{\partial z \partial \theta} \\
& +\frac{3 D_{50} l^{2}}{4 R^{2}} \frac{\partial^{3} W}{\partial z^{2} \partial \theta}+\frac{D_{50} l^{2}}{4 R^{4}} \frac{\partial^{3} W}{\partial \theta^{3}} \\
& -\frac{\left(D_{10}+\frac{D_{50} l^{2}}{4 R^{2}}+D_{50}\right)}{R^{2}} \frac{\partial W}{\partial \theta} \\
& -\frac{D_{51} l^{2}}{4 R^{3}} \frac{\partial^{4} \psi_{z}}{\partial z \partial \theta^{3}}-\frac{D_{51} l^{2}}{4 R} \frac{\partial^{4} \psi_{z}}{\partial z^{3} \partial \theta} \\
& -\frac{D_{50}}{R}\left(\frac{l^{2}}{4 R^{2}}+1\right) \psi_{\theta} \\
& -\frac{1}{R}\left(\frac{D_{50} l^{2}}{2 R}+D_{51}+D_{31}+\frac{D_{51} l^{2}}{4 R^{2}}\right) \frac{\partial^{2} \psi_{z}}{\partial z \partial \theta} \\
& -\frac{1}{R^{2}}\left(\frac{D_{50} l^{2}}{4 R}+D_{11}\right) \frac{\partial^{2} \psi_{\theta}}{\partial \theta^{2}}+\frac{D_{51} l^{2}}{4 R^{2}} \frac{\partial^{4} \psi_{\theta}}{\partial z^{2} \partial \theta^{2}} \\
& +\frac{D_{51} l^{2}}{4} \frac{\partial^{4} \psi_{\theta}}{\partial z^{4}}-\left(\frac{3 D_{50} l^{2}}{4 R}+D_{51}+\frac{D_{51} l^{2}}{4 R^{2}}\right) \frac{\partial^{2} \psi_{\theta}}{\partial z^{2}} \\
& +I_{10} \frac{\partial^{2} V}{\partial t^{2}}+\sum_{i=1}^{N_{s}}-\frac{1}{R^{\prime 2}}\left(R_{10}+\frac{R_{50} l^{2}}{4 R^{\prime 2}}\right) \frac{\partial^{2} V}{\partial \theta^{2}} \delta\left(z-z_{i}\right)
\end{aligned}
$$




$$
\begin{aligned}
& +\frac{R_{50}}{R^{\prime 2}}\left(\frac{l^{2}}{4 R^{\prime 2}}+1\right) V \delta\left(z-z_{i}\right) \\
& +\frac{R_{50} l^{2}}{4 R^{\prime 4}} \frac{\partial^{3} W}{\partial \theta^{3}} \delta\left(z-z_{i}\right) \\
& -\frac{\left(R_{10}+\frac{R_{50} l^{2}}{4 R^{\prime 2}}+R_{50}\right)}{R^{\prime 2}} \frac{\partial W}{\partial \theta} \delta\left(z-z_{i}\right) \\
& -\frac{R_{50} l^{2}}{4 R^{\prime 3}} \frac{\partial^{2} \psi_{\theta}}{\partial \theta^{2}} \delta\left(z-z_{i}\right) \\
& -\frac{R_{50}}{R^{\prime}}\left(\frac{l^{2}}{4 R^{\prime 2}}+1\right) \psi_{\theta} \delta\left(z-z_{i}\right) \\
& +J_{10} \frac{\partial^{2} V}{\partial t^{2}} \delta\left(z-z_{i}\right) \\
& \delta W: \frac{D_{50} l^{2}}{4} \frac{\partial^{4} W}{\partial z^{4}}-D_{50}\left(\frac{l^{2}}{4 R^{2}}+1\right) \frac{\partial^{2} W}{\partial z^{2}} \\
& +\frac{D_{50} l^{2}}{2 R^{2}} \frac{\partial^{4} W}{\partial z^{2} \partial \theta^{2}}+\frac{D_{10}}{R^{2}} W+\frac{D_{50} l^{2}}{4 R^{4}} \frac{\partial^{4} W}{\partial \theta^{4}} \\
& -\frac{D_{50}}{R^{2}}\left(\frac{l^{2}}{4 R^{2}}+1\right) \frac{\partial^{2} W}{\partial \theta^{2}}+\frac{D_{30}}{R} \frac{\partial U}{\partial z} \\
& +\frac{D_{50} l^{2}}{2 R^{3}} \frac{\partial^{3} U}{\partial z \partial \theta^{2}}-\frac{3 D_{50} l^{2}}{4 R^{2}} \frac{\partial^{3} V}{\partial z^{2} \partial \theta} \\
& -\frac{D_{50} l^{2}}{4 R^{4}} \frac{\partial^{3} V}{\partial \theta^{3}}+\frac{1}{R^{2}}\left(D_{10}+\frac{D_{50} l^{2}}{4 R^{2}}+D_{50}\right) \frac{\partial V}{\partial \theta} \\
& -\frac{D_{50} l^{2}}{4 R^{2}} \frac{\partial^{3} \psi_{z}}{\partial z \partial \theta^{2}}-\frac{D_{50} l^{2}}{4} \frac{\partial^{3} \psi_{z}}{\partial z^{3}} \\
& +\left(\frac{D_{50} l^{2}}{4 R^{2}}-D_{50}+\frac{D_{31}}{R}\right) \frac{\partial \psi_{z}}{\partial z}-\frac{D_{50} l^{2}}{4 R^{3}} \frac{\partial^{3} \psi_{\theta}}{\partial \theta^{3}} \\
& -\frac{1}{R}\left(\frac{D_{50} l^{2}}{4 R^{2}}+D_{50}-\frac{D_{11}}{R}\right) \frac{\partial \psi_{\theta}}{\partial \theta} \\
& -\frac{1}{2 R}\left(\frac{D_{50} l^{2}}{2}+\frac{D_{51} l^{2}}{R}\right) \frac{\partial^{3} \psi_{\theta}}{\partial z^{2} \partial \theta}+I_{10} \frac{\partial^{2} W}{\partial t^{2}} \\
& +\sum_{i=1}^{N_{s}} \frac{R_{50} l^{2}}{4 R^{\prime 4}} \frac{\partial^{4} W}{\partial \theta^{4}} \delta\left(z-z_{i}\right) \\
& -\frac{R_{50}}{{R^{\prime}}^{2}}\left(\frac{l^{2}}{4 R^{\prime 2}}+1\right) \frac{\partial^{2} W}{\partial \theta^{2}} \delta\left(z-z_{i}\right) \\
& +\frac{R_{10}}{{R^{\prime}}^{2}} W \delta\left(z-z_{i}\right)-\frac{R_{50} l^{2}}{4 R^{\prime 4}} \frac{\partial^{3} V}{\partial \theta^{3}} \delta\left(z-z_{i}\right) \\
& -\frac{R_{50} l^{2}}{4 R^{\prime 3}} \frac{\partial^{3} \psi_{\theta}}{\partial \theta^{3}} \delta\left(z-z_{i}\right)
\end{aligned}
$$$$
\begin{aligned}
& +\frac{1}{R^{\prime 2}}\left(R_{10}+\frac{R_{50} l^{2}}{4 R^{\prime 2}}+R_{50}\right) \frac{\partial V}{\partial \theta} \delta\left(z-z_{i}\right) \\
& -\frac{1}{R^{\prime}}\left(\frac{R_{50} l^{2}}{4 R^{\prime 2}}+R_{50}\right) \frac{\partial \psi_{\theta}}{\partial \theta} \delta\left(z-z_{i}\right) \\
& +J_{10} \frac{\partial^{2} W}{\partial t^{2}} \delta\left(z-z_{i}\right)
\end{aligned}
$$$$
\delta \psi_{z}:-\left(D_{12}+\frac{D_{50} l^{2}}{4}\right) \frac{\partial^{2} \psi_{z}}{\partial z^{2}}+\frac{D_{52} l^{2}}{4 R^{2}} \frac{\partial^{4} \psi_{z}}{\partial z^{2} \partial \theta^{2}}
$$$$
+\frac{D_{52} l^{2}}{4 R^{4}} \frac{\partial^{4} \psi_{z}}{\partial \theta^{4}}+D_{50}\left(\frac{l^{2}}{4 R^{2}}+1\right) \psi_{z}
$$$$
-\frac{1}{R^{2}}\left(D_{50} l^{2}+D_{52}-\frac{D_{51} l^{2}}{2 R}\right) \frac{\partial^{2} \psi_{z}}{\partial \theta^{2}}
$$$$
-\frac{1}{R}\left(D_{32}+D_{52}-\frac{3 D_{50} l^{2}}{4}+\frac{D_{51} l^{2}}{2 R}\right) \frac{\partial^{2} \psi_{\theta}}{\partial z \partial \theta}
$$$$
-\frac{D_{52} l^{2}}{4 R^{3}} \frac{\partial^{4} \psi_{\theta}}{\partial z \partial \theta^{3}}-\frac{D_{52} l^{2}}{4 R} \frac{\partial^{4} \psi_{\theta}}{\partial z^{3} \partial \theta}
$$$$
+\frac{1}{R^{2}}\left(\frac{5 D_{50} l^{2}}{4 R}-D_{51}\right) \frac{\partial^{2} U}{\partial \theta^{2}}-D_{11} \frac{\partial^{2} U}{\partial z^{2}}
$$$$
+\frac{D_{51} l^{2}}{4 R^{4}} \frac{\partial^{4} U}{\partial \theta^{4}}+\frac{D_{51} l^{2}}{4 R^{2}} \frac{\partial^{4} U}{\partial z^{2} \partial \theta^{2}}-\frac{D_{51} l^{2}}{4 R^{3}} \frac{\partial^{4} V}{\partial z \partial \theta^{3}}
$$$$
-\frac{D_{51} l^{2}}{4 R} \frac{\partial^{4} V}{\partial z^{3} \partial \theta}+\frac{D_{50} l^{2}}{4} \frac{\partial^{3} W}{\partial \theta^{3}}
$$$$
-\frac{1}{R}\left(\frac{D_{50} l^{2}}{2 R}+D_{31}+D_{51}+\frac{D_{51} l^{2}}{4 R^{2}}\right) \frac{\partial^{2} V}{\partial z \partial \theta}
$$$$
-\left(\frac{D_{50} l^{2}}{4 R^{2}}-D_{50}+\frac{D_{31}}{R}\right) \frac{\partial W}{\partial z}
$$$$
+\frac{D_{50} l^{2}}{4 R^{2}} \frac{\partial^{3} W}{\partial z \partial \theta^{2}}+I_{12} \frac{\partial^{2} \psi_{z}}{\partial t^{2}}
$$$$
+\sum_{i=1}^{N_{s}} \frac{R_{52} l^{2}}{4 R^{\prime 4}} \frac{\partial^{4} \psi_{z}}{\partial \theta^{4}} \delta\left(z-z_{i}\right)
$$$$
-\frac{1}{R^{\prime 2}}\left(R_{50} l^{2}+R_{52}\right) \frac{\partial^{2} \psi_{z}}{\partial \theta^{2}} \delta\left(z-z_{i}\right)
$$$$
+R_{50}\left(\frac{l^{2}}{4 R^{\prime 2}}+1\right) \psi_{z} \delta\left(z-z_{i}\right)
$$$$
+\frac{5 R_{50} l^{2}}{4 R^{\prime 3}} \frac{\partial^{2} U}{\partial \theta^{2}} \delta\left(z-z_{i}\right)+\frac{R_{50} l^{2}}{4} \frac{\partial^{3} W}{\partial \theta^{3}} \delta\left(z-z_{i}\right)
$$$$
+J_{12} \frac{\partial^{2} \psi_{z}}{\partial t^{2}} \delta\left(z-z_{i}\right),
$$ 


$$
\begin{aligned}
& \delta \psi_{\theta}: \frac{D_{52} l^{2}}{4} \frac{\partial^{4} \psi_{\theta}}{\partial z^{4}} \\
& -\left(D_{52}+D_{50} l^{2}+\frac{D_{52} l^{2}}{2 R^{2}}+\frac{D_{51} l^{2}}{2 R}\right) \frac{\partial^{2} \psi_{\theta}}{\partial z^{2}} \\
& +\frac{D_{52} l^{2}}{4 R^{2}} \frac{\partial^{4} \psi_{\theta}}{\partial z^{2} \partial \theta^{2}}+\frac{D_{51} l^{2}}{4} \frac{\partial^{4} V}{\partial z^{4}} \\
& -\frac{1}{R^{2}}\left(D_{12}+\frac{D_{50} l^{2}}{4}\right) \frac{\partial^{2} \psi_{\theta}}{\partial \theta^{2}} \\
& -\frac{1}{R}\left(D_{32}+D_{52}-\frac{3 D_{50} l^{2}}{4}+\frac{D_{51} l^{2}}{2 R}\right) \frac{\partial^{2} \psi_{z}}{\partial z \partial \theta} \\
& -\frac{D_{52} l^{2}}{4 R^{3}} \frac{\partial^{4} \psi_{z}}{\partial z \partial \theta^{3}}-\frac{D_{52} l^{2}}{4 R} \frac{\partial^{4} \psi_{z}}{\partial z^{3} \partial \theta} \\
& +D_{50}\left(\frac{l^{2}}{4 R^{2}}+1\right) \psi_{\theta}-\frac{D_{51} l^{2}}{4 R^{3}} \frac{\partial^{4} U}{\partial z \partial \theta^{3}} \\
& -\frac{D_{51} l^{2}}{4 R} \frac{\partial^{4} U}{\partial z^{3} \partial \theta} \\
& -\frac{1}{R}\left(\frac{D_{50} l^{2}}{4 R}+D_{31}+D_{51}-\frac{D_{51} l^{2}}{2 R^{2}}\right) \frac{\partial^{2} U}{\partial z \partial \theta} \\
& -\frac{1}{R^{2}}\left(\frac{D_{50} l^{2}}{4 R}+D_{11}\right) \frac{\partial^{2} V}{\partial \theta^{2}} \\
& -\left(\frac{3 D_{50} l^{2}}{4 R}+D_{51}+\frac{D_{51} l^{2}}{4 R^{2}}\right) \frac{\partial^{2} V}{\partial z^{2}} \\
& -\frac{D_{50}}{R}\left(\frac{l^{2}}{4 R^{2}}+1\right) V+\frac{D_{51} l^{2}}{2 R^{2}} \frac{\partial^{4} V}{\partial z^{2} \partial \theta^{2}} \\
& +\frac{1}{R}\left(\frac{D_{50} l^{2}}{4}+\frac{D_{51} l^{2}}{2 R}\right) \frac{\partial^{3} W}{\partial z^{2} \partial \theta}+\frac{D_{50} l^{2}}{4 R^{3}} \frac{\partial^{3} W}{\partial \theta^{3}} \\
& +\frac{1}{R}\left(\frac{D_{50} l^{2}}{4 R^{2}}+D_{50}-\frac{D_{11}}{R}\right) \frac{\partial W}{\partial \theta}+I_{12} \frac{\partial^{2} \psi_{\theta}}{\partial t^{2}} \\
& +\sum_{i=1}^{N_{s}}-\frac{1}{{R^{\prime}}^{2}}\left(R_{12}+\frac{R_{50} l^{2}}{4}\right) \frac{\partial^{2} \psi_{\theta}}{\partial \theta^{2}} \delta\left(z-z_{i}\right) \\
& -\frac{R_{50} l^{2}}{4 R^{\prime 3}} \frac{\partial^{2} V}{\partial \theta^{2}} \delta\left(z-z_{i}\right) \\
& -\frac{R_{50}}{R^{\prime}}\left(\frac{l^{2}}{4 R^{\prime 2}}+1\right) V \delta\left(z-z_{i}\right) \\
& +\frac{R_{50} l^{2}}{4 R^{\prime 3}} \frac{\partial^{3} W}{\partial \theta^{3}} \delta\left(z-z_{i}\right) \\
& +\frac{1}{R^{\prime}}\left(\frac{R_{50} l^{2}}{4 R^{\prime 2}}+R_{50}\right) \frac{\partial W}{\partial \theta} \delta\left(z-z_{i}\right)
\end{aligned}
$$

$$
\begin{aligned}
& +R_{50}\left(\frac{l^{2}}{4 R^{\prime 2}}+1\right) \psi_{\theta} \delta\left(z-z_{i}\right) \\
& +J_{12} \frac{\partial^{2} \psi_{\theta}}{\partial t^{2}} \delta\left(z-z_{i}\right)
\end{aligned}
$$

Dirac function shows the place of ring stiffener in the cylindrical shell. Boundary conditions of the cylindrical shell for $z=$ constant are as follows:

$$
\begin{aligned}
& \int_{\theta}\left[D_{10} \frac{\partial U}{\partial z}-\frac{D_{50} l^{2}}{4 R^{2}} \frac{\partial^{3} U}{\partial z \partial \theta^{2}}+\frac{D_{50} l^{2}}{4 R} \frac{\partial^{3} V}{\partial z^{2} \partial \theta}\right. \\
& +\frac{1}{R}\left(D_{30}+\frac{D_{50} l^{2}}{4 R^{2}}\right) \frac{\partial V}{\partial \theta}+\frac{D_{30}}{R} W \\
& -\frac{D_{50} l^{2}}{4 R^{3}} \frac{\partial^{2} W}{\partial \theta^{2}}+\frac{1}{R}\left(D_{31}-\frac{D_{50} l^{2}}{4 R}\right) \frac{\partial \psi_{\theta}}{\partial \theta} \\
& +D_{11} \frac{\partial \psi_{z}}{\partial z}+\frac{D_{51} l^{2}}{4 R} \frac{\partial^{3} \psi_{\theta}}{\partial z^{2} \partial \theta}-\frac{D_{51} l^{2}}{4 R^{2}} \frac{\partial^{3} \psi_{z}}{\partial z \partial \theta^{2}} \\
& +\sum_{i=1}^{N_{s}} \frac{1}{R^{\prime}}\left(R_{30}+\frac{R_{50} l^{2}}{4{R^{\prime}}^{2}}\right) \frac{\partial V}{\partial \theta} \delta\left(z-z_{i}\right) \\
& +\frac{R_{30}}{R^{\prime}} W \delta\left(z-z_{i}\right)-\frac{R_{50} l^{2}}{4 R^{\prime 3}} \frac{\partial^{2} W}{\partial \theta^{2}} \delta\left(z-z_{i}\right) \\
& \left.+\frac{1}{R^{\prime}}\left(R_{31}-\frac{R_{50} l^{2}}{4 R^{\prime}}\right) \frac{\partial \psi_{\theta}}{\partial \theta} \delta\left(z-z_{i}\right)\right]\left.d \theta\right|_{z=0, L}=0 \\
& \text { or }\left.\delta U\right|_{z=0, L}=0 \text {, } \\
& \int_{\theta}\left[-\frac{D_{50} l^{2}}{4} \frac{\partial^{3} V}{\partial z^{3}}+\left(D_{50}+\frac{3 D_{50} l^{2}}{4 R^{2}}\right) \frac{\partial V}{\partial z}\right. \\
& -\frac{D_{50} l^{2}}{4 R^{2}} \frac{\partial^{3} V}{\partial z \partial \theta^{2}}+\frac{D_{50} l^{2}}{4 R} \frac{\partial^{3} U}{\partial z^{2} \partial \theta}+\frac{D_{50} l^{2}}{4 R^{3}} \frac{\partial^{3} U}{\partial \theta^{3}} \\
& +\frac{D_{50}}{R}\left(1-\frac{l^{2}}{2 R^{2}}\right) \frac{\partial U}{\partial \theta}-\frac{D_{50} l^{2}}{4 R^{2}} \frac{\partial^{2} W}{\partial z \partial \theta} \\
& +\frac{1}{R}\left(D_{51}+\frac{3 D_{50} l^{2}}{4 R}\right) \frac{\partial \psi_{z}}{\partial \theta}+\frac{D_{51} l^{2}}{4 R^{3}} \frac{\partial^{3} \psi_{z}}{\partial \theta^{3}} \\
& +\frac{D_{51} l^{2}}{4 R} \frac{\partial^{3} \psi_{z}}{\partial z^{2} \partial \theta}+\left(D_{51}+\frac{3 D_{50} l^{2}}{4 R}+\frac{D_{51} l^{2}}{2 R^{2}}\right) \frac{\partial \psi_{\theta}}{\partial z} \\
& -\frac{D_{51} l^{2}}{4 R^{2}} \frac{\partial^{3} \psi_{\theta}}{\partial z \partial \theta^{2}}-\frac{D_{51} l^{2}}{4} \frac{\partial^{3} \psi_{\theta}}{\partial z^{3}} \\
& +\sum_{i=1}^{N_{s}} \frac{R_{50} l^{2}}{4 R^{\prime 3}} \frac{\partial^{3} U}{\partial \theta^{3}} \delta\left(z-z_{i}\right)
\end{aligned}
$$




$$
\begin{aligned}
& +\frac{R_{50}}{R^{\prime}}\left(1-\frac{l^{2}}{2 R^{\prime 2}}\right) \frac{\partial U}{\partial \theta} \delta\left(z-z_{i}\right) \\
& \text { or }\left.\delta W\right|_{z=0, L}=0 \text {, } \\
& +\frac{1}{R^{\prime}}\left(R_{51}+\frac{3 R_{50} l^{2}}{4 R^{\prime}}\right) \frac{\partial \psi_{z}}{\partial \theta} \delta\left(z-z_{i}\right) \\
& \int_{\theta}\left[\frac{D_{50} l^{2}}{4} \frac{\partial^{2} W}{\partial z^{2}}-\frac{D_{50} l^{2}}{4 R^{2}} \frac{\partial^{2} W}{\partial \theta^{2}}\right. \\
& \left.+\frac{R_{51} l^{2}}{4 R^{\prime 3}} \frac{\partial^{3} \psi_{z}}{\partial \theta^{3}} \delta\left(z-z_{i}\right)\right]\left.d \theta\right|_{z=0, L}=0 \\
& +\frac{D_{50} l^{2}}{4 R^{2}} \frac{\partial V}{\partial \theta}+\frac{D_{50} l^{2}}{4 R} \frac{\partial \psi_{\theta}}{\partial \theta}-\frac{D_{50} l^{2}}{4} \frac{\partial \psi_{z}}{\partial z} \\
& +\sum_{i=1}^{N_{s}}-\frac{R_{50} l^{2}}{4 R^{\prime 2}} \frac{\partial^{2} W}{\partial \theta^{2}} \delta\left(z-z_{i}\right) \\
& +\frac{R_{50} l^{2}}{4 R^{\prime 2}} \frac{\partial V}{\partial \theta} \delta\left(z-z_{i}\right) \\
& \left.+\frac{R_{50} l^{2}}{4 R^{\prime}} \frac{\partial \psi_{\theta}}{\partial \theta} \delta\left(z-z_{i}\right)\right]\left.d \theta\right|_{z=0, L}=0 \\
& -\frac{D_{51} l^{2}}{4 R} \frac{\partial^{2} \psi_{z}}{\partial z \partial \theta}+\sum_{i=1}^{N_{s}} \frac{R_{50} l^{2}}{4{R^{\prime}}^{2}} V \delta\left(z-z_{i}\right) \\
& -\frac{R_{50} l^{2}}{4 R^{\prime 2}} \frac{\partial W}{\partial \theta} \delta\left(z-z_{i}\right) \\
& \left.-\frac{R_{50} l^{2}}{4 R^{\prime}} \psi_{\theta} \delta\left(z-z_{i}\right)\right]\left.d \theta\right|_{z=0, L}=0 \\
& \text { or }\left.\delta\left(\frac{\partial V}{\partial z}\right)\right|_{z=0, L}=0 \\
& \int_{\theta}\left[-\frac{D_{50} l^{2}}{4} \frac{\partial^{3} W}{\partial z^{3}}+D_{50}\left(1+\frac{l^{2}}{4 R^{2}}\right) \frac{\partial W}{\partial z}\right. \\
& -\frac{3 D_{50} l^{2}}{4 R^{2}} \frac{\partial^{3} W}{\partial z \partial \theta^{2}}-\frac{3 D_{50} l^{2}}{4 R^{3}} \frac{\partial^{2} U}{\partial \theta^{2}}+\frac{D_{50} l^{2}}{R^{2}} \frac{\partial^{2} V}{\partial z \partial \theta} \\
& +D_{50}\left(1-\frac{l^{2}}{4 R^{2}}\right) \psi_{z}+\frac{D_{50} l^{2}}{4} \frac{\partial^{2} \psi_{z}}{\partial z^{2}} \\
& +\frac{1}{R^{2}}\left(\frac{D_{50} l^{2}}{2}-\frac{D_{51} l^{2}}{4 R}\right) \frac{\partial^{2} \psi_{z}}{\partial \theta^{2}} \\
& +\frac{1}{R}\left(\frac{D_{50} l^{2}}{4}+\frac{3 D_{51} l^{2}}{4 R}\right) \frac{\partial^{2} \psi_{\theta}}{\partial z \partial \theta} \\
& +\sum_{i=1}^{N_{s}}-\frac{3 R_{50} l^{2}}{4 R^{\prime 3}} \frac{\partial^{2} U}{\partial \theta^{2}} \delta\left(z-z_{i}\right) \\
& +\frac{1}{{R^{\prime}}^{2}}\left(\frac{R_{50} l^{2}}{2}-\frac{R_{51} l^{2}}{4 R^{\prime}}\right) \frac{\partial^{2} \psi_{z}}{\partial \theta^{2}} \delta\left(z-z_{i}\right) \\
& \left.+R_{50}\left(1-\frac{l^{2}}{4 R^{\prime 2}}\right) \psi_{z} \delta\left(z-z_{i}\right)\right]\left.d \theta\right|_{z=0, L}=0 \\
& \text { or }\left.\delta\left(\frac{\partial W}{\partial z}\right)\right|_{z=0, L}=0 \\
& \int_{\theta}\left[-\frac{D_{50} l^{2}}{4} \frac{\partial^{2} W}{\partial z^{2}}+\frac{1}{R^{2}}\left(\frac{D_{50} l^{2}}{4}-\frac{D_{51} l^{2}}{4 R}\right) \frac{\partial^{2} W}{\partial \theta^{2}}\right. \\
& +\frac{1}{R}\left(D_{32}-\frac{D_{50} l^{2}}{4}-\frac{D_{51} l^{2}}{4 R}\right) \frac{\partial \psi_{\theta}}{\partial \theta} \\
& +\left(D_{12}+\frac{D_{50} l^{2}}{4}\right) \frac{\partial \psi_{z}}{\partial z} \\
& +\frac{1}{R}\left(D_{31}-\frac{D_{50} l^{2}}{4 R}+\frac{D_{51} l^{2}}{4 R^{2}}\right) \frac{\partial V}{\partial \theta} \\
& -\frac{D_{52} l^{2}}{4 R^{2}} \frac{\partial^{3} \psi_{z}}{\partial z \partial \theta^{2}}+\frac{D_{52} l^{2}}{4 R} \frac{\partial^{3} \psi_{\theta}}{\partial z^{2} \partial \theta} \\
& -\frac{D_{51} l^{2}}{4 R^{2}} \frac{\partial^{3} U}{\partial z \partial \theta^{2}}+\frac{D_{51} l^{2}}{4 R} \frac{\partial^{3} V}{\partial z^{2} \partial \theta} \\
& +\sum_{i=1}^{N_{s}} \frac{1}{R^{\prime}}\left(R_{32}-\frac{R_{50} l^{2}}{4}-\frac{R_{51} l^{2}}{4 R^{\prime}}\right) \frac{\partial \psi_{\theta}}{\partial \theta} \delta\left(z-z_{i}\right) \\
& +\frac{1}{R^{\prime}}\left(R_{31}-\frac{R_{50} l^{2}}{4 R^{\prime}}+\frac{R_{51} l^{2}}{4 R^{\prime 2}}\right) \frac{\partial V}{\partial \theta} \delta\left(z-z_{i}\right) \\
& \left.+\frac{1}{{R^{\prime}}^{2}}\left(\frac{R_{50} l^{2}}{4}-\frac{R_{51} l^{2}}{4 R^{\prime}}\right) \frac{\partial^{2} W}{\partial \theta^{2}} \delta\left(z-z_{i}\right)\right]\left.d \theta\right|_{z=0, L}=0 \\
& \text { or }\left.\delta \psi_{z}\right|_{z=0, L}=0 \text {, }
\end{aligned}
$$




$$
\begin{aligned}
& +\frac{1}{R}\left(D_{51}+\frac{D_{50} l^{2}}{2 R}-\frac{D_{51} l^{2}}{2 R^{2}}\right) \frac{\partial U}{\partial \theta} \\
& +\frac{D_{51} l^{2}}{4 R^{3}} \frac{\partial^{3} U}{\partial \theta^{3}}+\frac{D_{51} l^{2}}{4 R} \frac{\partial^{3} U}{\partial z^{2} \partial \theta} \\
& +\left(D_{52}+D_{52} l^{2}+\frac{D_{52} l^{2}}{2 R^{2}}+\frac{D_{51} l^{2}}{4 R}\right) \frac{\partial \psi_{\theta}}{\partial z} \\
& +\frac{1}{R}\left(D_{52}-\frac{D_{50} l^{2}}{2}+\frac{3 D_{51} l^{2}}{4 R}\right) \frac{\partial \psi_{z}}{\partial \theta} \\
& +\frac{D_{52} l^{2}}{4 R} \frac{\partial^{3} \psi_{z}}{\partial z^{2} \partial \theta}+\left(D_{51}+\frac{D_{51} l^{2}}{4 R^{2}}+\frac{D_{50} l^{2}}{2 R}\right) \frac{\partial V}{\partial z} \\
& -\frac{D_{51} l^{2}}{4 R^{2}} \frac{\partial^{3} V}{\partial z \partial \theta^{2}}-\frac{D_{51} l^{2}}{4} \frac{\partial^{3} V}{\partial z^{3}}-\frac{D_{52} l^{2}}{4 R^{2}} \frac{\partial^{3} \psi_{\theta}}{\partial z \partial \theta^{2}} \\
& +\frac{D_{52} l^{2}}{4 R^{3}} \frac{\partial^{3} \psi_{z}}{\partial \theta^{3}}-\frac{D_{52} l^{2}}{4} \frac{\partial^{3} \psi_{\theta}}{\partial z^{3}} \\
& +\sum_{i=1}^{N_{s}} \frac{R_{51} l^{2}}{4 R^{\prime 3}} \frac{\partial^{3} U}{\partial \theta^{3}} \delta\left(z-z_{i}\right) \\
& +\frac{1}{R^{\prime}}\left(R_{51}+\frac{R_{50} l^{2}}{2 R^{\prime}}-\frac{R_{51} l^{2}}{2 R^{\prime 2}}\right) \frac{\partial U}{\partial \theta} \delta\left(z-z_{i}\right) \\
& +\frac{R_{52} l^{2}}{4 R^{\prime 3}} \frac{\partial^{3} \psi_{z}}{\partial \theta^{3}} \delta\left(z-z_{i}\right)+\frac{1}{R^{\prime}}\left(R_{52}-\frac{R_{50} l^{2}}{2}\right. \\
& \left.\left.+\frac{3 R_{51} l^{2}}{4 R^{\prime}}\right) \frac{\partial \psi_{z}}{\partial \theta} \delta\left(z-z_{i}\right)\right]\left.d \theta\right|_{z=0, L}=0, \\
& \text { or }\left.\delta \psi_{\theta}\right|_{z=0, L}=0 \text {, } \\
& \int_{\theta}\left[-\frac{D_{52} l^{2}}{4 R} \frac{\partial^{2} \psi_{z}}{\partial z \partial \theta}+\frac{D_{52} l^{2}}{4} \frac{\partial^{2} \psi_{\theta}}{\partial z^{2}}+\frac{D_{51} l^{2}}{4 R^{2}} V\right. \\
& -\frac{D_{51} l^{2}}{4 R} \frac{\partial^{2} U}{\partial z \partial \theta}+\frac{D_{51} l^{2}}{4} \frac{\partial^{2} V}{\partial z^{2}}-\frac{D_{51} l^{2}}{4 R^{2}} \frac{\partial W}{\partial \theta} \\
& -\frac{D_{51} l^{2}}{4 R} \psi_{\theta}+\sum_{i=1}^{N_{s}} \frac{R_{51} l^{2}}{4 R^{\prime 2}} V \delta\left(z-z_{i}\right) \\
& -\frac{R_{51} l^{2}}{4 R^{\prime 2}} \frac{\partial W}{\partial \theta} \delta\left(z-z_{i}\right) \\
& \left.-\frac{R_{51} l^{2}}{4 R^{\prime}} \psi_{\theta} \delta\left(z-z_{i}\right)\right]\left.d \theta\right|_{z=0, L}=0 \\
& \text { or }\left.\delta\left(\frac{\partial \psi_{\theta}}{\partial z}\right)\right|_{z=0, L}=0 \text {. }
\end{aligned}
$$

Boundary conditions of the cylindrical shell for $\theta=$ constant are as follows:

$$
\begin{aligned}
& \int_{z}\left[-\frac{D_{50} l^{2}}{4 R^{4}} \frac{\partial^{3} U}{\partial \theta^{3}}+\frac{1}{R^{2}}\left(D_{50}+\frac{D_{50} l^{2}}{R^{2}}\right) \frac{\partial U}{\partial \theta}\right. \\
& -\frac{D_{50} l^{2}}{4 R^{2}} \frac{\partial^{3} U}{\partial z^{2} \partial \theta}+\frac{D_{50} l^{2}}{4 R^{3}} \frac{\partial^{3} V}{\partial z \partial \theta^{2}} \\
& +\frac{1}{R}\left(D_{50}-\frac{D_{50} l^{2}}{4 R^{2}}\right) \frac{\partial V}{\partial z}+\frac{D_{50} l^{2}}{4 R} \frac{\partial^{3} V}{\partial z^{3}} \\
& +\frac{D_{50} l^{2}}{2 R^{3}} \frac{\partial^{2} W}{\partial z \partial \theta}-\frac{1}{R^{2}}\left(\frac{5 D_{50} l^{2}}{4 R}-D_{51}\right) \frac{\partial \psi_{z}}{\partial \theta} \\
& +\frac{1}{R}\left(D_{51}-\frac{D_{51} l^{2}}{2 R^{2}}+\frac{D_{50} l^{2}}{4 R}\right) \frac{-\partial \psi_{\theta}}{\partial z} \\
& +\frac{D_{51} l^{2}}{4 R^{3}} \frac{\partial^{3} \psi_{\theta}}{\partial z \partial \theta^{2}}-\frac{D_{51} l^{2}}{4 R^{4}} \frac{\partial^{3} \psi_{z}}{\partial \theta^{3}} \\
& +\frac{D_{51} l^{2}}{4 R} \frac{\partial^{3} \psi_{\theta}}{\partial z^{3}}-\frac{D_{51} l^{2}}{4 R^{2}} \frac{\partial^{3} \psi_{z}}{\partial z^{2} \partial \theta} \\
& +\sum_{i=1}^{N_{s}}-\frac{R_{50} l^{2}}{4 R^{\prime}} \frac{\partial^{3} U}{\partial \theta^{3}} \delta\left(z-z_{i}\right) \\
& +\frac{1}{{R^{\prime 2}}^{2}}\left(R_{50}+\frac{R_{50} l^{2}}{{R^{\prime}}^{2}}\right) \frac{\partial U}{\partial \theta} \delta\left(z-z_{i}\right) \\
& -\frac{1}{{R^{\prime}}^{2}}\left(\frac{5 R_{50} l^{2}}{4 R^{\prime}}-R_{51}\right) \frac{\partial \psi_{z}}{\partial \theta} \delta\left(z-z_{i}\right) \\
& \left.-\frac{R_{51} l^{2}}{4 R^{\prime}} \frac{\partial^{3} \psi_{z}}{\partial \theta^{3}} \delta\left(z-z_{i}\right)\right]\left.d z\right|_{\theta=0, \theta_{0}}=0, \\
& \text { or }\left.\delta U\right|_{\theta=0, \theta_{0}}=0 \text {, } \\
& \int_{z}\left[\frac{D_{50} l^{2}}{4 R^{4}} \frac{\partial^{2} U}{\partial \theta^{2}}-\frac{D_{50} l^{2}}{4 R^{3}} \frac{\partial^{2} V}{\partial z \partial \theta}-\frac{D_{50} l^{2}}{4 R^{3}} \frac{\partial W}{\partial z}\right. \\
& +\frac{D_{50} l^{2}}{4 R^{3}} \psi_{z}-\frac{D_{51} l^{2}}{4 R^{3}} \frac{\partial^{2} \psi_{\theta}}{\partial z \partial \theta}+\frac{D_{51} l^{2}}{4 R^{4}} \frac{\partial^{2} \psi_{z}}{\partial \theta^{2}} \\
& +\sum_{i=1}^{N_{s}} \frac{R_{50} l^{2}}{4 R^{\prime 4}} \frac{\partial^{2} U}{\partial \theta^{2}} \delta\left(z-z_{i}\right)+\frac{R_{50} l^{2}}{4 R^{\prime 3}} \psi_{z} \delta\left(z-z_{i}\right) \\
& \left.+\frac{R_{51} l^{2}}{4 R^{\prime 4}} \frac{\partial^{2} \psi_{z}}{\partial \theta^{2}} \delta\left(z-z_{i}\right)\right]\left.d z\right|_{\theta=0, \theta_{0}}=0 \\
& \text { or }\left.\delta\left(\frac{\partial U}{\partial \theta}\right)\right|_{z=0, \theta_{0}}=0,
\end{aligned}
$$




$$
\begin{aligned}
\int_{z}\left[\frac{1}{R^{2}}\left(\frac{D_{50} l^{2}}{4 R^{2}}+D_{10}\right) \frac{\partial V}{\partial \theta}-\frac{D_{50} l^{2}}{4 R^{2}} \frac{\partial^{3} V}{\partial z^{2} \partial \theta}\right. \\
+\frac{D_{50} l^{2}}{4 R^{3}} \frac{\partial^{3} U}{\partial z \partial \theta^{2}}+\frac{D_{30}}{R} \frac{\partial U}{\partial z}+\frac{D_{10}}{R^{2}} W \\
\quad-\frac{D_{50} l^{2}}{4 R^{4}} \frac{\partial^{2} W}{\partial \theta^{2}}+\frac{1}{R^{2}}\left(\frac{D_{50} l^{2}}{4 R}+D_{11}\right) \frac{\partial \psi_{\theta}}{\partial \theta} \\
+\frac{D_{31}}{R} \frac{\partial \psi_{z}}{\partial z}-\frac{D_{51} l^{2}}{4 R^{2}} \frac{\partial^{3} \psi_{\theta}}{\partial z^{2} \partial \theta}+\frac{D_{51} l^{2}}{4 R^{3}} \frac{\partial^{3} \psi_{z}}{\partial z \partial \theta^{2}} \\
+\sum_{i=1}^{N_{s}} \frac{1}{R^{\prime 2}}\left(\frac{R_{50} l^{2}}{4 R^{\prime 2}}+R_{10}\right) \frac{\partial V}{\partial \theta} \delta\left(z-z_{i}\right) \\
+\frac{R_{10}}{{R^{\prime 2}}^{2}} W \delta\left(z-z_{i}\right)-\frac{R_{50} l^{2}}{4 R^{\prime}} \frac{\partial^{2} W}{\partial \theta^{2}} \delta\left(z-z_{i}\right) \\
\left.+\frac{1}{{R^{\prime 2}}^{2}}\left(\frac{R_{50} l^{2}}{4 R^{\prime}}+R_{11}\right) \frac{\partial \psi_{\theta}}{\partial \theta} \delta\left(z-z_{i}\right)\right]\left.d z\right|_{\theta=0, \theta_{0}}=0
\end{aligned}
$$$$
\text { or }\left.\delta V\right|_{\theta=0, \theta_{0}}=0 \text {, }
$$

$$
\begin{aligned}
\int_{z}[ & -\frac{D_{50} l^{2}}{4 R^{4}} \frac{\partial^{3} W}{\partial \theta^{3}}+\frac{D_{50} l^{2}}{R^{2}}\left(1+\frac{l^{2}}{4 R^{2}}\right) \frac{\partial W}{\partial \theta} \\
& -\frac{3 D_{50} l^{2}}{4 R^{2}} \frac{\partial^{3} W}{\partial z^{2} \partial \theta}+\frac{3 D_{50} l^{2}}{4 R^{2}} \frac{\partial^{2} V}{\partial z^{2}}+\frac{D_{50} l^{2}}{4 R^{4}} \frac{\partial^{2} V}{\partial \theta^{2}} \\
& -\frac{D_{50}}{R^{2}}\left(1+\frac{l^{2}}{4 R^{2}}\right) V-\frac{D_{50} l^{2}}{4 R^{3}} \frac{\partial^{2} U}{\partial z \partial \theta} \\
& +\frac{l^{2}}{4 R^{2}}\left(D_{50}+\frac{D_{51}}{R}\right) \frac{\partial^{2} \psi_{z}}{\partial z \partial \theta}+\frac{D_{50}}{R}\left(1+\frac{l^{2}}{4 R^{2}}\right) \psi_{\theta} \\
& +\frac{D_{50} l^{2}}{4 R^{3}} \frac{\partial^{2} \psi_{\theta}}{\partial \theta^{2}}+\frac{1}{R}\left(\frac{D_{50} l^{2}}{2}+\frac{D_{51} l^{2}}{4 R}\right) \frac{\partial^{2} \psi_{\theta}}{\partial z^{2}} \\
& +\sum_{i=1}^{N_{s}} \frac{R_{50} l^{2}}{4 R^{\prime}} \frac{\partial^{3} W}{\partial \theta^{3}} \delta\left(z-z_{i}\right)+\frac{R_{50} l^{2}}{4 R^{4}} \frac{\partial^{2} V}{\partial \theta^{2}} \delta\left(z-z_{i}\right) \\
& -\frac{R_{50}}{R^{\prime 2}}\left(1+\frac{l^{2}}{4 R^{\prime 2}}\right) V \delta\left(z-z_{i}\right) \\
& +\frac{R_{50}}{R^{\prime}}\left(1+\frac{l^{2}}{4 R^{\prime 2}}\right) \psi_{\theta} \delta\left(z-z_{i}\right) \\
& +\frac{R_{50} l^{2}}{4 R^{\prime 3}} \frac{\partial^{2} \psi_{\theta}}{\partial \theta^{2}} \delta\left(z-z_{i}\right) \\
& \left.+\frac{R_{50} l^{2}}{R^{\prime 2}}\left(1+\frac{l^{2}}{4 R^{\prime 2}}\right) \frac{\partial W}{\partial \theta} \delta\left(z-z_{i}\right)\right]\left.d z\right|_{\theta=0, \theta_{0}}=0 \\
&
\end{aligned}
$$

$$
\begin{aligned}
& \text { or }\left.\delta W\right|_{\theta=0, \theta_{0}}=0 \text {, } \\
& \int_{z}\left[-\frac{D_{50} l^{2}}{4 R^{4}} \frac{\partial V}{\partial \theta}-\frac{D_{50} l^{2}}{4 R^{2}} \frac{\partial^{2} W}{\partial z^{2}}+\frac{D_{50} l^{2}}{4 R^{4}} \frac{\partial^{2} W}{\partial \theta^{2}}\right. \\
& +\frac{D_{50} l^{2}}{4 R^{2}} \frac{\partial \psi_{z}}{\partial z}-\frac{D_{50} l^{2}}{4 R^{3}} \frac{\partial \psi_{\theta}}{\partial \theta} \\
& +\sum_{i=1}^{N_{s}} \frac{R_{50} l^{2}}{4 R^{\prime 4}} \frac{\partial^{2} W}{\partial \theta^{2}} \delta\left(z-z_{i}\right)-\frac{R_{50} l^{2}}{4 R^{\prime 4}} \frac{\partial V}{\partial \theta} \delta\left(z-z_{i}\right) \\
& \left.-\frac{R_{50} l^{2}}{4 R^{\prime 3}} \frac{\partial \psi_{\theta}}{\partial \theta} \delta\left(z-z_{i}\right)\right]\left.d z\right|_{\theta=0, \theta_{0}}=0, \\
& \text { or }\left.\delta\left(\frac{\partial W}{\partial \theta}\right)\right|_{\theta=0, \theta_{0}}=0
\end{aligned}
$$$$
\int_{z}\left[\frac{1}{R^{2}}\left(-\frac{D_{50} l^{2}}{R}+D_{51}\right) \frac{\partial U}{\partial \theta}\right.
$$$$
+\frac{1}{R}\left(\frac{D_{50} l^{2}}{2 R}+\frac{D_{51} l^{2}}{4 R^{2}}+D_{51}\right) \frac{\partial V}{\partial z}-\frac{D_{50} l^{2}}{2 R^{2}} \frac{\partial^{2} W}{\partial z \partial \theta}
$$$$
+\frac{1}{R^{2}}\left(-\frac{D_{51} l^{2}}{4 R}+D_{52}+D_{50} l^{2}\right) \frac{\partial \psi_{z}}{\partial \theta}
$$$$
-\frac{D_{52} l^{2}}{4 R^{4}} \frac{\partial^{3} \psi_{z}}{\partial \theta^{3}}+\frac{D_{52} l^{2}}{4 R^{3}} \frac{\partial^{3} \psi_{\theta}}{\partial z \partial \theta^{2}}+\frac{D_{51} l^{2}}{4 R^{3}} \frac{\partial^{3} V}{\partial z \partial \theta^{2}}
$$$$
+\frac{D_{51} l^{2}}{4 R} \frac{\partial^{3} V}{\partial z^{3}}-\frac{D_{51} l^{2}}{4 R^{2}} \frac{\partial^{3} U}{\partial z^{2} \partial \theta}-\frac{D_{51} l^{2}}{4 R^{4}} \frac{\partial^{3} U}{\partial \theta^{3}}
$$$$
+\frac{1}{R}\left(-\frac{D_{50} l^{2}}{2}+\frac{D_{51} l^{2}}{4 R}+D_{52}\right) \frac{\partial \psi_{\theta}}{\partial z}
$$$$
-\frac{D_{52} l^{2}}{4 R^{2}} \frac{\partial^{3} \psi_{z}}{\partial z^{2} \partial \theta}+\frac{D_{52} l^{2}}{4 R} \frac{\partial^{3} \psi_{\theta}}{\partial z^{3}}
$$$$
+\sum_{i=1}^{N_{s}} \frac{1}{R^{\prime 2}}\left(-\frac{R_{50} l^{2}}{R^{\prime}}+R_{51}\right) \frac{\partial U}{\partial \theta} \delta\left(z-z_{i}\right)
$$$$
+\frac{1}{{R^{\prime}}^{2}}\left(-\frac{R_{51} l^{2}}{4 R^{\prime}}+R_{52}+R_{50} l^{2}\right) \frac{\partial \psi_{z}}{\partial \theta} \delta\left(z-z_{i}\right)
$$$$
-\frac{R_{52} l^{2}}{4 R^{\prime}} \frac{\partial^{3} \psi_{z}}{\partial \theta^{3}} \delta\left(z-z_{i}\right)
$$$$
\left.-\frac{R_{51} l^{2}}{4 R^{\prime 4}} \frac{\partial^{3} U}{\partial \mathrm{heta}^{3}} \delta\left(z-z_{i}\right)\right]\left.d z\right|_{\theta=0, \theta_{0}}=0,
$$$$
\text { or }\left.\delta \psi_{z}\right|_{\theta=0, \theta_{0}}=0 \text {, }
$$ 


$$
\begin{aligned}
& \int_{z}\left[\frac{D_{52} l^{2}}{4 R^{4}} \frac{\partial^{2} \psi_{z}}{\partial \theta^{2}}-\frac{D_{52} l^{2}}{4 R^{3}} \frac{\partial^{2} \psi_{\theta}}{\partial z \partial \theta}-\frac{D_{51} l^{2}}{4 R^{3}} \frac{\partial^{2} V}{\partial z \partial \theta}\right. \\
& +\frac{D_{51} l^{2}}{4 R^{4}} \frac{\partial^{2} U}{\partial \theta^{2}}-\frac{D_{51} l^{2}}{4 R^{3}} \frac{\partial W}{\partial z}+\frac{D_{51} l^{2}}{4 R^{3}} \psi_{z} \\
& +\sum_{i=1}^{N_{s}} \frac{R_{52} l^{2}}{4 R^{\prime 4}} \frac{\partial^{2} \psi_{z}}{\partial \theta^{2}} \delta\left(z-z_{i}\right)+\frac{R_{51} l^{2}}{4 R^{\prime}} \frac{\partial^{2} U}{\partial \theta^{2}} \delta\left(z-z_{i}\right) \\
& \left.+\frac{R_{51} l^{2}}{4 R^{\prime 3}} \psi_{z} \delta\left(z-z_{i}\right)\right]\left.d z\right|_{\theta=0, \theta_{0}}=0 \\
& \text { or }\left.\delta\left(\frac{\partial \psi_{z}}{\partial \theta}\right)\right|_{\theta=0, \theta_{0}}=0 \\
& \int_{z} \frac{1}{R^{2}}\left(-\frac{D_{50} l^{2}}{4 R}+D_{11}\right) \frac{\partial V}{\partial \theta} \\
& +\frac{l^{2}}{4 R}\left(-\frac{D_{51}}{R}+D_{50}\right) \frac{\partial^{2} W}{\partial z^{2}} \\
& +\frac{1}{R}\left(-\frac{D_{50} l^{2}}{4}+D_{32}\right) \frac{\partial \psi_{z}}{\partial z}+\frac{D_{52} l^{2}}{4 R^{3}} \frac{\partial^{3} \psi_{z}}{\partial z \partial \theta^{2}} \\
& +\frac{1}{R^{2}}\left(\frac{D_{50} l^{2}}{4}+D_{12}\right) \frac{\partial \psi_{\theta}}{\partial \theta}-\frac{D_{52} l^{2}}{4 R^{2}} \frac{\partial^{3} \psi_{\theta}}{\partial z^{2} \partial \theta} \\
& -\frac{D_{50} l^{2}}{4 R^{3}} \frac{\partial^{2} W}{\partial \theta^{2}}+\frac{D_{11}}{R^{2}} W+\frac{D_{31}}{R} \frac{\partial U}{\partial z} \\
& -\frac{D_{51} l^{2}}{4 R^{2}} \frac{\partial^{3} V}{\partial z^{2} \partial \theta}+\frac{D_{51} l^{2}}{4 R^{3}} \frac{\partial^{3} U}{\partial z \partial \theta^{2}}+\frac{D_{51} l^{2}}{4 R^{2}} \frac{\partial \psi_{z}}{\partial z} \\
& +\sum_{i=1}^{N_{s}}-\frac{R_{50} l^{2}}{4 R^{\prime}} \frac{\partial^{2} W}{\partial \theta^{2}} \delta\left(z-z_{i}\right) \\
& \frac{1}{{R^{\prime}}^{2}}\left(-\frac{R_{50} l^{2}}{4 R^{\prime}}+R_{11}\right) \frac{\partial V}{\partial \theta} \delta\left(z-z_{i}\right) \\
& +\frac{1}{R^{\prime 2}}\left(\frac{R_{50} l^{2}}{4}+R_{12}\right) \frac{\partial \psi_{\theta}}{\partial \theta} \delta\left(z-z_{i}\right)
\end{aligned}
$$

$$
\begin{aligned}
& +\left.\frac{R_{11}}{R^{\prime 2}} W \delta\left(z-z_{i}\right) d z\right|_{\theta=0, \theta_{0}}=0, \\
& \text { or }\left.\quad \delta \psi_{\theta}\right|_{\theta=0, \theta_{0}}=0 .
\end{aligned}
$$

Eqs. (A.2) to (A.6) are equations of motion of FGM stiffened micro cylinder, and Eqs. (A.7) to (A.22) are classical and non-classical boundary conditions of this structure derived by the modified couple stress theory and the first-order shear deformation theory using Hamilton's principle.

\section{Biographies}

Siavash Jabbarian was born in Tehran in 1991. By achieving a high rank in the university entrance exam, he decided to study Mechanical Engineering in Sharif University of Technology. He obtained his bachelor degree with good grades and worked on non-destructive testing equipment using Eddy current methods with prof. Ahmadian. He continued his Master education in Applied Mechanics and concentrated his studies on micro and nano structure's dynamics. As for his thesis, he worked with professor Ahmadian on the vibration of the micro cylinders on the base of modified couple stress theory and investigated the vibrational behavior of micro cylinder that is stiffened by micro rings.

Mohammd Taghi Ahmadian received a PhD degree from the Department of Mechanical Engineering in the University of Kansas, USA in 1986. From 1984 to 1985, he served as an Assistant Professor in the University of Missouri, and from 1985 to 1988, he was an Assistant Professor at the Department of Mechanical Engineering at the University of Kansas at Lawrence. He started working as an Assistant Professor in Sharif university of Technology from 1989; at present, he is a Professor in the School of Mechanical Engineering at Sharif University of Technology, Tehran, Iran. His research interests include cellular biomechanics, soft tissue modeling, strength of material, and advanced finite-element method. 